\title{
BEYOND ARBITRAGE: "GOOD-DEAL" ASSET PRICE BOUNDS IN INCOMPLETE MARKETS
}

John H. Cochrane Jesús Saá-Requejo

Working Paper 5489

\section{NATIONAL BUREAU OF ECONOMIC RESEARCH 1050 Massachusetts Avenue \\ Cambridge, MA 02138 \\ March 1996}

We thank the NSF and the Graduate School of Business for research support. We thank George Constantinides, Kent Daniel, Doug Diamond, Lars Hansen, Ken Singleton, seminar participants and especially Yacine Aït-Sahalia for many helpful discussions. This paper is part of NBER's research program in Asset Pricing. Any opinions expressed are those of the authors and not those of the National Bureau of Economic Research.

(C) 1996 by John H. Cochrane and Jesús Saá-Requejo. All rights reserved. Short sections of text, not to exceed two paragraphs, may be quoted without explicit permission provided that full credit, including $\odot$ notice, is given to the source. 


\title{
BEYOND ARBITRAGE: "GOOD-DEAL" \\ ASSET PRICE BOUNDS IN \\ INCOMPLETE MARKETS
}

\begin{abstract}
It is often useful to price assets and other random payoffs by reference to other observed prices rather than construct full-fledged economic asset pricing models. This approach breaks down if one cannot find a perfect replicating portfolio. We impose weak economic restrictions to derive usefully tight bounds on asset prices in this situation. The bounds basically rule out high Sharpe ratios - "good deals" - as well as arbitrage opportunities. We present the method of calculation, we extend it to a multiperiod context by finding a recursive solution, and we apply it to option pricing examples including the Black-Scholes setup with infrequent trading, and a model with stochastic stock volatility and a varying riskfree rate.
\end{abstract}

John H. Cochrane

Graduate School of Business

University of Chicago

1101 East 58th Street

Chicago, IL 60637

and NBER
Jesús Saá-Requejo

Graduate School of Business

University of Chicago

1101 East 58th Street

Chicago, IL 60637 


\section{Contents}

1 Introduction $\quad 1$

2 Stochastic discount factors $\quad 6$

2.1 Definition, existence and interpretation . . . . . . . . . . . . 6

2.2 Discount factors and Sharpe ratios . . . . . . . . . . . . . . . 6

2.3 What value of the volatility constraint should we impose? . . . . . . . . 8

3 Price bounds with one trading period 12

3.1 Volatility constraint . . . . . . . . . . . . . . . 13

3.1.1 Alternative derivations; more intuition . . . . . . . . . . . 16

3.2 Adding the positive discount factor constraint . . . . . . . . . . . 18

3.3 Application to the Black-Scholes setup . . . . . . . . . . . . . . . . 21

3.3.1 Calculating moments from a lognormal model . . . . . . . . . . . . . 21

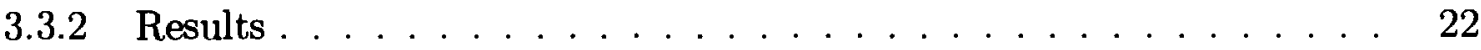

3.4 Price bounds with multiple options . . . . . . . . . . . . . . . . . . . . 24

3.4 .1 Results . . . . . . . . . . . . . . . . . 25

4 Deltas $\quad 26$

5 Price bounds with multiple trading periods - a recursive solution 29

5.1 A multiperiod multinomial application . . . . . . . . . . . . . . 31

5.1 .1 Results . . . . . . . . . . . . . . . . . . 33

6 Continuous time and missing assets $\quad 34$

6.1 Discount factors in continuous time . . . . . . . . . . . . . . 34

6.2 Deriving price bounds $\ldots \ldots \ldots \ldots$

6.2.1 Recursive and then differential statement of the problem . . . . . . 37

6.2 .2 Constraints . . . . . . . . . . . . . . . 38

6.2.3 Solutions: a characterization . . . . . . . . . . . . . . 40

6.2.4 Solution 1: a partial differential equation for the price bounds . . . . 43 
6.2.5 Solution 2: a special case in which we know the discount factor. . . . 44

6.3 Pricing a call option with stochastic stock volatility and interest rates . . . 45

6.4 A stochastic-volatility stochastic-interest-rate model . . . . . . . . . . 46

6.5 Results . . . . . . . . . . . . . . . . . . 47

$\begin{array}{llr}7 \text { Conclusions and Extensions } & 50\end{array}$

8 Appendix $\quad 52$

8.1 Evaluating expectations with positive discount factors and lognormal returns. 52

8.2 Multiple option integrals . . . . . . . . . . . . . . . . . 53

9 References. $\quad 55$ 


\section{Introduction}

The basic question of finance theory is how to value uncertain payoffs. The law of one price implies that we can always write a price as the expected discounted value of the payoff. It implies that there is a stochastic discount factor $m$ such that

$$
p_{t}=E_{t}\left(m_{t+1} x_{t+1}\right)
$$

where $p=$ price and $x=$ payoff. Hence, one can cast the issue as a search for the discount factor $m$. There are two basic approaches.

1) Construct economic models for discount factors that can price a wide class of payoffs. The consumption-based asset pricing model of Lucas (1978) Rubinstein (1976) Merton (1973a) and others is the canonical example of this approach. In simple versions of this model, one predicts that the price $p$ of any payoff $x$ is given by

$$
p_{t}=E_{t}\left(\delta \frac{u^{\prime}\left(c_{t+1}\right)}{u^{\prime}\left(c_{t}\right)} x_{t+1}\right)
$$

where $u=$ utility function, $c=$ consumption and $\delta=$ subjective discount factor. The Capital Asset Pricing Model and related models are special cases.

2) Extract information about a discount factor $m$ that correctly prices a given payoff from observations of the price of other assets, without worrying about the determinants of the other assets' prices. This is the idea behind the classic options pricing theory of Black and Scholes (1973) and Merton (1973b): determine the price of an option from the price of the underlying stock and a bond. It is the motivation for Ross' (1976) Arbitrage Pricing Theory: determine the risk premium (expected rate of return) of a given stock or portfolio from the risk premia of a few "factor" portfolios. This approach underlies many applications of asset-pricing theory to corporate finance. For example, one examines the returns on "like" securities to determine the "hurdle rates" for a specific project. Via its role in option pricing theory, this method is at the heart of many recent applications of finance theory to economics.

For many cases this is the right approach. One is not particularly interested in the economic determinants of discount factors and asset prices, and one desires tighter predictions for specific prices than the economic approach allows. 
However, it only works if the payoff in question can be exactly replicated by a portfolio of tradeable assets. If not, then discount factors exist that price the tradeable assets and generate any price for the payoff in question. In most applications one does not in fact have a perfect replicating portfolio. One only has an approximate hedge, a portfolio whose payoff is "near" or "like" the payoff in question.

We use a little economics to restrict the range of possible stochastic discount factors. These restrictions result in bounds on an asset price, given the prices of a set of basis assets that can be used as approximate hedges ${ }^{1}$. We require that the stochastic discount factor is positive, and we impose an upper bound on the volatility of the stochastic discount factor.

The positive discount factor constraint $m \geq 0$ results from nonsatiation or positive marginal utility. Used alone, it rules out arbitrage opportunities (positive payoffs with negative prices).

Following Hansen and Jagannathan (1991), the volatility of marginal utility is a robust quantity that one can learn about from a variety of data sources and thought experiments. An upper bound on plausible consumption volatility and risk aversion results in a useful upper bound on the volatility of marginal utility. Knowledge or priors about a Sharpe ratio (mean return to standard deviation) that would induce traders to buy any asset also implies an upper bound on discount factor volatility. Used alone, an upper bound on discount factor volatility rules out portfolios with high Sharpe ratios - good deals.

These discount factor restrictions mean that "small" (low-variance) payoffs must have small prices. Hence, if the residual or difference between the payoff in question and the payoff of the best approximate replicating portfolio is small (in a variance or $R^{2}$ sense) then the price must be close to the price of the best approximate replicating portfolio.

\section{Option pricing}

We apply these ideas to the pricing of options and other derivatives. The standard dynamic hedging arguments used to price options often break down. For example, one may not be able to trade continuously, or there may be state variables (stochastic volatility

\footnotetext{
${ }^{1}$ In this, our approach is similar to Constantinides (1994), who derives option pricing bounds from the weak restriction that the discount factor decreases monotonically with a state variable.
} 
and interest rate) that do not correspond to traded assets and hence whose risk prices are unobserved. This "incomplete markets" situation is pervasive: if options really could be perfectly and costlessly replicated by other liquid assets, it is unlikely that options would be traded in the first place! It is common to assume prices for missing securities and then pretend to price options by arbitrage arguments. Instead, we calculate good-deal bounds, using a set of approximate hedges that are actually available.

We start with a set of basis assets, whose prices and payoffs are observed and tradeablea stock and bond in the classic Black-Scholes (1973) - Merton (1973b) case, but an arbitrary set of assets including other options in general. Then, we maximize and minimize option prices by choice of stochastic discount factors that correctly price the basis assets and satisfy the positivity and volatility constraints. In equations, we solve problems like

$$
\underline{C}=\min _{\{m\}} E\left(m x^{c}\right) \text { s.t. } \mathbf{p}=E(m \mathbf{x}) ; m \geq 0 ; \sigma(m) \leq h / R^{f}
$$

where

$$
\begin{aligned}
\underline{C} & =\text { lower bound on option price } \\
m & =\text { discount factor, } \\
x^{c} & =\text { option payoff, } \\
\mathbf{p}, \mathbf{x} & =\text { price and payoffs of basis assets (vectors) } \\
h & =\text { prespecified volatility bound (Sharpe ratio units) } \\
R^{f} & =\text { risk free interest rate. }
\end{aligned}
$$

In this context, the no-arbitrage or positive discount factor constraint alone yields wellknown and typically quite wide arbitrage bounds for the option price. But the discount factors required to attain the arbitrage bounds are often unreasonable. For example, in order to attain the lower arbitrage bound $C \geq \max \left(0, S-K / R^{f}\right)(S=$ stock price, $K=$ strike price) for a European call option, marginal utility must be zero for all states of nature in which the option finishes out of the money. Attaining the upper arbitrage bound $C \leq S$ requires a discount factor that is only non-zero in the two states of nature with the most extreme stock prices. These are unlikely characterizations of anyone's marginal utility! The volatility constraint weeds out such arbitrage-free but still "unreasonable" discount factors and their corresponding option prices. 
Asset pricing in general and option pricing in particular are essentially multiperiod questions. One wants a discount factor process and option price bound functions (of state variables). To this end, we extend problem (1) to multiperiod contexts. We find a recursive solution, i.e. we show that the lower bound today solves the one period problem (1) with the lower bound tomorrow in the place of the option payoff $x^{c}$. This formulation makes the multiperiod problem computationally feasible; it leads to a partial differential equation for the bounds in a continuous time context.

Pricing methodologies are only important if they are usable. To this end, we show how to calculate price bounds in common option-pricing setups. We calculate call option price bounds in a Black-Scholes world with discrete trading opportunities; in a multiperiod, multinomial environment; and in a continuous-time environment with stochastic interest rates and stochastic volatility. Aït-Sahalia, Cochrane and Saá-Requejo (1996) use the same techniques to calculate bounds on the price of a yield cap in a singularity-free continuous time term structure model.

Good-deal bounds should be useful in many situations for which replication arguments such as option pricing formulas are used to think about one's own valuation or market valuations, but perfect replication is not possible. 1) A trader can use our bounds as "buy" and "sell" points in the search for "good deals" in asset markets (with the usual warning question why the market leaves good deals undiscovered). 2) A bank or other institution that seeks to market or synthesize a non-traded security can use good-deal bounds as bid and ask prices, using its target rates of return and risk exposure to define a good deal. 3) Gooddeal bounds can be used as economic measures of the accuracy of option pricing formulas. Arbitrage-based formulas predict no error, so that "measurement errors" in prices must be tacked on to the models. Our bounds tell us which option prices should lie close to arbitragebased formulas, and which can lie far from the predictions of those formulas, using an economic measure of distance. 4) Option pricing techniques are increasingly applied to "real options" in capital budgeting, investment with irreversibilities and policy questions. Our bounds provide a way to answer these questions that does not require perfect replication, but also does not require that we wait for the construction of completely specified economicallybased asset pricing models that can value any payoff. 5) Option pricing formulas are often used in risk assessment, to quantify the exposure of a position or institution to various risk 
factors. Obviously, a way to assess such risks without imposing unverifiable assumptions is useful.

Good-deal price bounds are not only interesting when they are small. When the bounds are large, they quantify our ignorance. They tell us that conventional option pricing approaches are particularly bad, and that the approximate hedges are in fact not very good. Large bounds force us to think about a price via its covariance with discount factors or (equivalently) risk-factor exposure rather than just try to use information in existing prices. 


\section{Stochastic discount factors}

\subsection{Definition, existence and interpretation}

A stochastic discount factor is a random variable $m$ that generates the price $p$ of every random payoff $x$ in a collection of payoffs by $p=E(m x)$. (Returns are payoffs with price 1 , excess returns are payoffs with price 0 .)

The marginal utility of any investor is a discount factor. For example, if an investor $i$ has a utility function $E \sum_{t} \delta^{t} u\left(c_{t}^{i}\right)$, then his first-order conditions for optimal consumption and portfolio choices give

$$
p_{t}=E_{t}\left(\delta \frac{u^{\prime}\left(c_{t+1}^{i}\right)}{u^{\prime}\left(c_{t}^{i}\right)} x_{t+1}\right) .
$$

Here, marginal utility growth $m_{t+1}=\delta u^{\prime}\left(c_{t+1}^{i}\right) / u^{\prime}\left(c_{t}^{i}\right)$ is a discount factor. (We omit time subscripts below when they are clear.) Pricing models like the CAPM or factor models can be expressed as linear models for the discount factor. The discount factor is also the change of measure in a risk-neutral probability or martingale measure asset pricing language, and is a set of contingent claims prices scaled by probabilities.

In frictionless markets, the law of one price (portfolios with identical payoffs must have the same price) holds if an only if a discount factor exists. The absence of arbitrage (any nonnegative payoff has a nonnegative price) holds if and only if a strictly positive discount factor exists. (Harrison and Kreps 1979.) We use these existence theorems: we search for positive discount factors as a simple way to enforce no arbitrage and the law of one price without having to construct portfolios.

\subsection{Discount factors and Sharpe ratios}

We impose constraints on the volatility of discount factors. Discount factor volatility is closely related to Sharpe ratios. The following two propositions clarify this connection. 
Proposition 1 The Sharpe ratio of any asset priced by a discount factor $m$ is less than or equal to the ratio of standard deviation to mean of that discount factor. Formally, if $0=E\left(m R^{e}\right)$ then

$$
\frac{\left|E\left(R^{e}\right)\right|}{\sigma\left(R^{e}\right)} \leq \frac{\sigma(m)}{E(m)}
$$

where $R^{e}$ denotes excess returns.

Proof:

$$
\begin{gathered}
0=E\left(m R^{e}\right)=E(m) E\left(R^{e}\right)+\rho_{m, R^{e}} \sigma(m) \sigma\left(R^{e}\right) \\
\frac{E\left(R^{e}\right)}{\sigma\left(R^{e}\right)}=-\rho_{m, R^{e}} \frac{\sigma(m)}{E(m)} .
\end{gathered}
$$

$\left|\rho_{m, R^{e}}\right| \leq 1$ gives the bound.

This proposition is just a reinterpretation of the Hansen-Jagannathan (1991) bound. Hansen and Jagannathan use it to learn about minimum discount factor volatility from Sharpe ratios; we use it to learn about maximum Sharpe ratios from discount factor volatility.

If markets are complete, then there is a unique discount factor, and we know there is a portfolio whose Sharpe ratio achieves the upper bound. If markets are incomplete, then there are many possible discount factors. The maximum attainable Sharpe ratio equals the minimum ratio of standard deviation to mean among all discount factors.

This proposition shows that the discount factor volatility constraint has a Sharpe ratio interpretation: if we impose $\sigma(m) / E(m) \leq h$ then no portfolio priced by $m$ can have Sharpe ratio greater than $h$.

An inequality version of the same logic helps us to find a value for the upper bound on discount factor volatility.

Proposition 2 If a trader will accept any investment with a Sharpe ratio greater than $h$, then the standard deviation / mean ratio of his marginal rate of substitution is less than $h$. Formally, if $R^{e}$ is an excess return and

$$
\frac{E\left(R^{e}\right)}{\sigma\left(R^{e}\right)} \geq h \text { implies } E\left(m R^{e}\right) \geq 0
$$

then we know that

$$
\frac{\sigma(m)}{E(m)} \leq h
$$


Proof. Using the covariance decomposition, we have

$$
\begin{gathered}
E(m) E\left(R^{e}\right)+\rho_{m, R^{e}} \sigma(m) \sigma\left(R^{e}\right) \geq 0 \\
-\rho_{m, R^{e}} \frac{\sigma(m)}{E(m)} \leq \frac{E\left(R^{e}\right)}{\sigma\left(R^{e}\right)}
\end{gathered}
$$

The tightest bound comes from a security with $\rho=-1$ and $E\left(R^{e}\right) / \sigma\left(R^{e}\right)=h$.

In the statement of the proposition, $E\left(m R^{e}\right)>0$ formalizes the idea that the trader "wants to buy" the high Sharpe ratio security or portfolio: the trader's valuation $E\left(m R^{e}\right)$ exceeds the market valuation, 0 . The Hansen-Jagannathan bound gives a lower bound on the variance of discount factors that price a collection of assets. Here, we find an upper bound by asking what assets are not priced-what assets a trader would want to buy if they were offered. We do not assume that a security with $|\rho|=1$ and $E\left(R^{e}\right) / \sigma\left(R^{e}\right)=h$ exists.

This proposition provides a way to measure and interpret an upper bound on discount factor volatility. If we ask a trader or investor "would you take any investment with Sharpe ratio greater than $h$ ?" and he responds affirmatively, then we know to impose the corresponding upper bound on the volatility of the discount factor. If the trader or investor also answers yes to "Would you take any arbitrage opportunity - any zero or negative cost position with a payoff that is nonnegative and might be positive?" or "Can you think of any state of the world in which you that you would throw money away?" we know in addition to impose $m>0$.

\subsection{What value of the volatility constraint should we impose?}

Three considerations (at least) are useful in thinking about plausible values for the volatility constraint: Sharpe ratios available in asset markets, Sharpe ratios that traders desire (that would induce them to trade), and observations on consumption volatility and risk aversion. The appropriate value also depends on the purpose to which the bound is put: If we want to establish trading rules or the value of an option to be synthesized for a client, we want to think about an individual trader's Sharpe ratio targets or marginal utility; if we want to establish a market valuation, we want to think about other traders' target Sharpe ratios or marginal utility volatility, or that of "representative agents." 
1) Market Sharpe ratios, traders and priors. The historical Sharpe ratio of market portfolios is about 0.5 ( $8 \%$ mean excess return, $16 \%$ standard deviation) on an annual basis. How much larger should a Sharpe ratio target be?

What Sharpe ratio are market participants willing to take? The mutual fund industry seems to search desperately for trading strategies that generate even a few percentage points above the market Sharpe ratio. Many institutions adopt explicit risk/return objectives, and traders receive explicit risk/return instructions. These objective are typically not huge multiples of the market portfolio Sharpe ratio. Below, we use a target Sharpe ratio of 1.0, twice the market Sharpe ratio, which seems if anything high in this context.

What Sharpe ratios are available? Equivalently, how inefficient is the market portfolio? If the market Sharpe ratio is 0.5 , are there portfolios that reliably yield Sharpe ratios of 1 or 2 (ex-ante, after transactions costs, and out of sample)? Many people state priors that the market portfolio is not so dramatically inefficient. For example, MacKinlay (1995) criticizes the Fama and French (1993) factor pricing model for using a factor mimicking portfolio with sample Sharpe ratios of about 1.0, which he finds unbelievably higher than the market portfolio Sharpe ratio.

Furthermore, if such high Sharpe ratios are available, why has the intensely competitive mutual fund industry not achieved them? Fund performance evaluations argue heatedly about whether the average fund achieves $1 \%$ above or $1 \%$ below index Sharpe ratios. The only way to reconcile this fact with a view that Sharpe ratios twice or more the market's are easily available is to argue that funds and their customers have objectives that differ dramatically from mean-variance efficiency. Other risk-factors matter a lot, investors desire portfolios that are multifactor efficient though dramatically mean-variance inefficient, and the market portfolio inherits these characteristics. Equivalently, one has to believe that funds and their customers are so concerned about tracking error relative to dramatically inefficient market indices that they forego investment opportunities with double or more the market Sharpe ratio. This view is hard to reconcile with many funds' colorful brochures that prominently advertize mean and variance. Furthermore, some funds should exist to serve investors who are not exposed to multifactor risks, and should regularly attain double the market Sharpe ratio. 
2) Utility functions and consumption. The discount factor is equal to marginal utility growth; using the standard power utility function, we have

$$
m_{t+1}=\delta\left(\frac{c_{t+1}}{c_{t}}\right)^{-\gamma}
$$

This relation holds for every investor, as well as for the "representative consumer" that emerges under preference restrictions or market completeness assumptions. Hence, one can think about plausible values for individual or aggregate consumption volatility and risk aversion. Table 1 presents some Sharpe ratio calculations based on a lognormal model for consumption growth and power utility. Aggregate nondurable consumption growth has a standard deviation of about $1 \%$ per year, so risk aversion less than about 10 suggests values for $h=\sigma(m) / E(m)$ less than 0.1 .

Table 1: Sharpe ratios generated by power utility and iid lognormal consumption growth.

\begin{tabular}{r|ccc} 
& \multicolumn{3}{|c}{$\sigma(\Delta c)$} \\
& 0.01 & 0.05 & 0.10 \\
\hline$\gamma=1$ & 0.0001 & 0.0025 & 0.010 \\
$\gamma=5$ & 0.0025 & 0.065 & 0.28 \\
$\gamma=10$ & 0.010 & 0.28 & 1.72
\end{tabular}

Each table entry is $\sigma(m) / E(m)=e^{\gamma^{2} \sigma^{2}}-1$. This formula holds for $m=e^{-\gamma \Delta c}$ and $\Delta c$ iid normal with variance $\sigma^{2}$.

Individual consumption growth is more volatile than the aggregate; still it is unlikely that many individuals (even traders) have a standard deviation of nondurable and services consumption growth (not durables purchases) as high as $10 \%$ per year. Unless one makes extreme assumptions about both consumption volatility and risk aversion, it is hard to generate Sharpe ratios above 0.3 .

Furthermore, our price bounds subject to a Sharpe ratio or discount factor volatility constraint make the worst-case assumption that marginal utility growth is perfectly correlated with a portfolio of asset payoffs. We could equivalently bound the standard deviation the projection of discount factors on the space of asset payoffs. $p=E(m x)$ holds if and only if $p=E[\operatorname{proj}(m \mid x) x]$. Idiosyncratic volatility is (by definition) orthogonal to asset payoffs. Therefore, even evidence for high individual discount factor volatility need not imply large Sharpe ratios on traded assets. 
In summary, utility functions and consumption data lead to numbers if anything lower than the market Sharpe ratio. This observation is just the equity premium puzzle ${ }^{2}$ (Mehra and Prescott 1985, see Cochrane and Hansen 1991 for a review).

\footnotetext{
${ }^{2}$ The equity premium literature has made many modifications to utility functions in an effort to boost discount factor volatility. Two deep problems hinder this effort. a) Devices such as habit-persistence to raise the variance of unexpected marginal utility, $\operatorname{var}_{t}\left(m_{t+1}\right)$ and hence equity premia typically raise the variance of expected marginal utility $\operatorname{var}\left[E_{t}\left(m_{t+1}\right)\right]$ producing the counterfactual prediction of a wildly varying risk free rate. b) Aggregate and individual consumption growth are not well correlated with asset returns; the projection of marginal utility growth on asset returns still has very little volatility.
} 


\section{Price bounds with one trading period}

We start with the simplest situation, one trading period. We show how to calculate price bounds, solutions to problem (1), and we apply our formulas to a Black-Scholes world.

Figure 1 describes the basic idea of our procedure and helps to define notation. $\mathbf{x}$ denotes a vector of time $T$ payoffs on a set of basis assets. $\mathbf{p}$ denotes their prices at the trading date, time 0. (Boldface indicates vectors.) $X \equiv\left\{\mathbf{c}^{\prime} \mathbf{x}, \mathbf{c} \in R^{N}\right\}$ denotes the space of payoffs of portfolios of the basis assets ${ }^{3}$. Though graphed as a line, $X$ is typically an infinitedimensional space. Discount factors $m$ generate the prices of payoffs $x$ in $X$ by an inner product $p=E(m x)$. Thus, the space of discount factors that generate the prices of payoffs in $X$ is a payoff $x^{*}$ in $X$, plus orthogonal components, as indicated in the figure. The figure shows the restricted range of positive discount factors and discount factors with constrained volatility. $x^{c}$ denotes the payoff that we wish to price. For example, $x^{c}=\max \left(S_{T}-K, 0\right)$ for a call option with strike price $\mathrm{K}$.

We presume a statistical model for the distribution of the payoffs $\mathbf{x}$, so that we can calculate first and second moments. We also assume that if it is not already traded, introducing the payoff $x^{c}$ does not alter the price or payoff distribution of basis assets. In general equilibrium, it might. This is a reasonable assumption for some purposes: valuing existing assets for the purposes of a small (relative to the market) trader, valuing a new security that a single institution wishes to synthesize for a few clients, or valuing a real option in a specific investment project. It is less implausible that introducing a new exchange-listed option or other security changes the distribution of underlying returns.

\footnotetext{
${ }^{3}$ Since we need second moments, they had better be defined, so we assume $E|\mathbf{x}|^{2}<\infty$. We also assume the basis assets have been pruned of redundant assets so $E \mathbf{x x}$ is nonsingular. Finally $X$ is a closed linear subspace of $L^{2}$, the space of all random variables with finite second moments, endowed with its usual inner product $\langle a \mid b\rangle=E(a b)$ and norm $|a|^{2}=\langle a \mid a\rangle$. We also limit consideration to discount factors $m \in L^{2}$. Following Hansen and Jagannathan (1991), these complete the technical assumptions required for our calculations.
} 


\subsection{Volatility constraint}

We start by ignoring the restriction that the discount factor must be positive, and just study the volatility constraint. In this case we can derive analytic solutions.

The lower bound problem is

$$
\underline{C}=\min _{\{m\}} E\left[m x^{c}\right] \text { s.t. } \mathbf{p}=E(m \mathbf{x}), \sigma(m) / E(m) \leq h .
$$

where $h$ is the desired maximum Sharpe ratio. The problem for the upper bound has max in the place of min.

It is convenient to express the volatility constraint as a second moment. We assume that one of the payoffs $x$ is a riskless return, so we can write $E(m)=1 / R^{f}$. Define

$$
A^{2} \equiv \frac{1+h^{2}}{R^{f^{2}}} .
$$

Our problem for the lower bound is now ${ }^{4}$

$$
\underline{C}=\min _{\{m\}} E\left[m x^{c}\right] \text { s.t. } E(m \mathbf{x})=\mathbf{p}, E\left(m^{2}\right) \leq A^{2} .
$$

Rather than solve this problem directly, we set up orthogonal decompositions of the option payoff and discount factor. The solution then pops out. (This solution strategy mirrors Hansen and Richard's 1987 characterization of the mean-variance frontier.) We extensively use the fact that $E(x y)=E[x \operatorname{proj}(y \mid X)]$.

First, we decompose the option payoff into two orthogonal components. Define the approximate hedge portfolio $\hat{x}^{c}$ by

$$
\hat{x}^{c} \equiv \operatorname{proj}\left(x^{c} \mid X\right)=E\left(x^{c} \mathbf{x}^{\prime}\right) E\left(\mathbf{x x}^{\prime}\right)^{-1} \mathbf{x}
$$

and define a corresponding residual $w$

$$
w \equiv x^{c}-\hat{x}^{c}=x^{c}-E\left(x^{c} \mathbf{x}^{\prime}\right) E\left(\mathbf{x x}^{\prime}\right)^{-1} \mathbf{x}
$$

$$
\begin{gathered}
\sigma^{2}(m)=E\left(m^{2}\right)-E(m)^{2} \leq h^{2} E(m)^{2} \\
E\left(m^{2}\right) \leq\left(1+h^{2}\right) E(m)^{2}=\frac{1+h^{2}}{R^{f 2}} \equiv A^{2}
\end{gathered}
$$


(see figure 1). Now we have the decomposition

$$
x^{c}=\hat{x}^{c}+w ; E\left(w \hat{x}^{c}\right)=0 .
$$

In fact, $w$ is orthogonal to any $x$ in $X, E(w \mathbf{x})=0$.

Second, we decompose discount factors. As in figure 1, any $m$ that prices the payoffs $\mathbf{x}$ can be expressed as a random variable $x^{*}=\operatorname{proj}(m \mid X)$ in $X$ that prices all $\mathbf{x}$, plus orthogonal random variables. We further divide the component of $m$ orthogonal to $X$ into a component in the direction of $w$ and an orthogonal component.

Proposition $3 A$ discount factor $m$ prices the basis assets, $\mathbf{p}=E(m \mathbf{x})$, if and only if it is of the form

$$
m=x^{*}+v w+\epsilon
$$

where

$$
x^{*}=\mathbf{p}^{\prime} E\left(\mathbf{x} \mathbf{x}^{\prime}\right)^{-1} \mathbf{x},
$$

$v$ is an arbitrary number, $\epsilon$ is any random variable with $E(\epsilon \mathbf{x})=0, E(\epsilon w)=0$, and $w$ is defined in equation (4).

Proof. If: $x^{*}$ satisfies $\mathbf{p}=E\left(x^{*} \mathbf{x}\right)$ by construction. $E(w \mathbf{x})=E(\epsilon \mathbf{x})=0$ so $\mathbf{p}=E\left[\left(x^{*}+v w+\epsilon\right) \mathbf{x}\right]$. Only if: For any $m$ that satisfies $\mathbf{p}=E(m \mathbf{x})$, we have $\operatorname{proj}(m \mid \mathbf{x})=E\left(m \mathbf{x}^{\prime}\right) E\left(\mathbf{x} \mathbf{x}^{\prime}\right)^{-1} \mathbf{x}$ by the OLS projection formula, and $\operatorname{proj}(m \mid \mathbf{x})=$ $\mathbf{p}^{\prime} E\left(\mathbf{x x}^{\prime}\right)^{-1} \mathbf{x}=x^{*}$ by the assumption that $\mathbf{p}=E(m \mathbf{x})$. We define a residual $\delta=m-x^{*}$. By construction, $E(\delta \mathbf{x})=0$. Thus, any discount factor $m$ can be represented as $m=x^{*}+\delta$. Projecting $\delta$ on $w$ and defining $\epsilon$ as the residual again, we obtain $m=x^{*}+v w+\epsilon$.

This proposition makes our minimization easy, since we can choose $v$ and $\epsilon$ rather than choose the random variable $m$ directly, with a Lagrange multiplier on the pricing constraint. We will quickly see that we want to choose $\epsilon=0$, so we are reduced to the choice of a single number $v$. 
Proposition 4 The discount factor that generates the lower option price bound defined by equation (3) is given by

$$
\underline{m}=x^{*}-\underline{v} w
$$

and the bound is

$$
\underline{C}=E\left(x^{*} x^{c}\right)-\underline{v} E\left(w^{2}\right)
$$

where

$$
\underline{v}=\sqrt{\frac{A^{2}-E\left(x^{* 2}\right)}{E\left(w^{2}\right)}}
$$

The formulas for the upper bound are given by a + sign in front of the square root terms.

Proof: We substitute for $m$ from equation (5) to express the volatility constraint as

$$
E\left(m^{2}\right)=E\left(x^{* 2}\right)+v^{2} E\left(w^{2}\right)+E\left(\epsilon^{2}\right) \leq A^{2}
$$

and to express the objective as

$$
E\left[m x^{c}\right]=E\left(x^{*} x^{c}\right)+v E\left(w x^{c}\right)+E\left(\epsilon x^{c}\right)=E\left(x^{*} x^{c}\right)+v E\left(w^{2}\right)
$$

$\left(E\left(w x^{c}\right)=E\left(w^{2}\right)\right.$ follows from the definition of $w$ above or from the geometry of figure 1.)

The problem now reduces to the choice of $v$ and $\epsilon$. Since $\epsilon$ only appears in the inequality constraint, we choose $\epsilon=0$. Now we have a linear objective subject to a quadratic constraint, and one parameter $v$ to choose. So long as $w \neq 0$ so $E\left(w^{2}\right) \neq 0$, the volatility constraint binds and we simply pick $v$ to satisfy the constraint, leading to equation (9). Equations (7) and (8) follow by substituting $\underline{v}$ in equation (5) and the objective, equation (10).

The Sharpe ratio bound must be greater than that generated by the basis assets, $A^{2} \geq E\left(x^{* 2}\right)=\mathbf{p}^{\prime} E\left(\mathbf{x x}^{\prime}\right)^{-1} \mathbf{p}$, of course, or there is no discount factor that even satisfies the constraints.

The first term in equation (8) is the price of the near-hedge portfolio ${ }^{5}$,

$$
E\left(x^{*} x^{c}\right)=E\left[x^{*} \hat{x}^{c}\right]=E\left[m \hat{x}^{c}\right]
$$

\footnotetext{
${ }^{5}$ Again, we use the fact that $E(x y)=E[x \operatorname{proj}(y \mid X)]$.
} 
for any $m$ that prices basis assets. This is the component of the option price that we can deduce by the law of one price. The second term is the largest possible price of the residual $w$ consistent with the volatility bound on $m$ :

$$
\underline{v} E\left(w^{2}\right)=E(\underline{v} w w)=E\left[\left(x^{*}+\underline{v} w\right) w\right]=E[\underline{m} w]
$$

The price of the residual depends (by sign) on whether we search for an upper or lower bound, on the difference between the Sharpe ratio bound and that available from the basis assets $A^{2}-E\left(x^{* 2}\right)$, and on the size of the residual $E\left(w^{2}\right)$. The closer the option payoff to the basis payoffs, or the smaller $E\left(w^{2}\right)$, the tighter the bounds.

For explicit calculations it is useful to substitute the definitions of $x^{*}$ and $w$ in equation (8) to obtain

$$
\underline{C}=\mathbf{p}^{\prime} E\left(\mathbf{x} \mathbf{x}^{\prime}\right)^{-1} E\left(\mathbf{x} x^{c}\right)-\sqrt{A^{2}-\mathbf{p}^{\prime} E\left(\mathbf{x} \mathbf{x}^{\prime}\right)^{-1} \mathbf{p}} \sqrt{E\left(x^{c 2}\right)-E\left(x^{c} \mathbf{x}^{\prime}\right) E\left(\mathbf{x} \mathbf{x}^{\prime}\right)^{-1} E\left(\mathbf{x} x^{c}\right)}
$$

The upper bound $\bar{C}$ is the same formula with a + sign in front of the square root. This formula is much less pretty, but it shows explicitly how to calculate the option price bound from a model for the second moments of $\mathbf{x}, x^{c}$.

This formula also emphasizes one important difference between our results and noarbitrage option pricing: good-deal bounds depend on the driving processes-on the actual rather than just risk-neutral probabilities. The Sharpe ratio is defined relative to actual probabilities, so they must matter. In the limit that pricing by arbitrage is possible, the dependence on actual probabilities vanishes, but only in that limit.

\subsubsection{Alternative derivations; more intuition}

First, we can relate the size of the price bounds to the $R^{2}$ of a regression of the option payoff on the basis asset payoffs. From equation (4) or the geometry of figure 1 we have

$$
E\left(x^{c 2}\right)=E\left[\hat{x}^{c 2}\right]+E\left(w^{2}\right)
$$

Hence, we can substitute $R^{2}$ for $E\left(w^{2}\right)$ via

$$
R^{2} \equiv \frac{E\left(\hat{x}^{c 2}\right)}{E\left(x^{c 2}\right)}=1-\frac{E\left(w^{2}\right)}{E\left(x^{c 2}\right)}
$$


We can then write the size of the bounds as

$$
\bar{C}-\underline{C}=2 \sqrt{E\left(x^{c 2}\right)} \sqrt{A^{2}-E\left(x^{* 2}\right)} \sqrt{1-R^{2}}
$$

Thus, the size of the bounds, scaled by the size of the option payoff, is directly related to the $R^{2}$ measure of how "close" the option payoff is to the basis payoff space. Again, payoffs that have better approximate hedges will have tighter bounds.

Second, we can approach the problem by explicitly bounding the price of the residual. Write

$$
C=E\left(m x^{c}\right)=E\left[m\left(\hat{x}^{c}+w\right)\right]=E\left(m \hat{x}^{c}\right)+E(m w)
$$

We know the first term; it is the price of the near-hedge portfolio $\hat{x}^{c}$, as given by equation (11). Thus, the issue is the price of the residual $E(m w)$. Using the orthogonal decomposition of $m$, we have

$$
E(m w)=E\left[\left(m-x^{*}\right) w\right]=\operatorname{cov}\left[\left(m-x^{*}\right), w\right]=\rho \sigma\left(m-x^{*}\right) \sigma(w) .
$$

(The first equality exploits $E(w \mathbf{x})=0$, the second uses $E(w)=0$ since there is a risk free rate.) Since $|\rho| \leq 1$, we bound the price of the residual by

$$
|E(m w)| \leq \sigma\left(m-x^{*}\right) \sigma(w) .
$$

This expression is the same as our previous bound. We can write

$$
\begin{aligned}
\sigma\left(m-x^{*}\right)= & \sqrt{E\left[\left(m-x^{*}\right)^{2}\right]-\left[E\left(m-x^{*}\right)\right]^{2}}=\sqrt{E\left(m^{2}\right)-E\left(x^{* 2}\right)} \\
& =\sqrt{A^{2}-E\left(x^{* 2}\right)}=\sqrt{\sigma^{2}(m)-\sigma^{2}\left(x^{*}\right)}
\end{aligned}
$$

(we use $E(m)=E\left(x^{*}\right)$ with a risk free rate).

This approach gives additional intuition. The price of the residual $w$ is the central question anytime one wants to price one payoff from the prices of another set of assets. The residual's price is $p(w)=E(m w)$, and $E(m w)=\operatorname{cov}(m, w)$ when there is a risk free rate. In classic option pricing, the residual $w$ is zero so we know its price must be zero, with no further discount factor restrictions. Here we note $|\operatorname{cov}(m, w)| \leq \sigma(m) \sigma(w)$. Hence, if $\sigma(w)$ is small, a bound on the volatility of $\sigma(m)$ can restrict the price of the residual $w$. We improve on this latter calculation by "using up" some of the allowed volatility of $m$ on the basis assets, giving $\sqrt{\sigma^{2}(m)-\sigma^{2}\left(x^{*}\right)}$ rather than $\sigma(m)$. 
As this discussion makes clear, the piece of potential information we do not use is correlations of the discount factor with asset payoffs. We take the worst case assumption that the correlation is \pm 1 . Therefore, if one wants to do better than good deal bounds, one has to include such covariance or correlation information.

In many asset pricing situations (for example, most stock pricing), the residual is large. Even restricting their variance, discount factors differ importantly on the price of a security in

question; $\sqrt{\sigma^{2}(m)-\sigma^{2}\left(x^{*}\right)} \sigma(w)$ is large. One must therefore bring in the extra structure of explicit discount factor models such as the consumption-based model or CAPM to calculate and restrict correlations.

Third, we can also solve the dual problem, finding the minimum volatility of the discount factor (and hence maximum Sharpe ratio) for any given option price. This problem has the same first-order conditions as our problem. Following Hansen and Jagannathan (1991), the volatility-minimizing $m$ is a linear function of basis and option payoffs, with second moment given by

$$
A^{2}=E\left(m^{2}\right)=\left[\begin{array}{ll}
C & \mathbf{p}^{\prime}
\end{array}\right] E\left[\begin{array}{cc}
x^{c 2} & x^{c} \mathbf{x}^{\prime} \\
x^{c} \mathbf{x} & \mathbf{x x}^{\prime}
\end{array}\right]^{-1}\left[\begin{array}{l}
C \\
\mathbf{p}
\end{array}\right]
$$

If we solve equation (12) for $C$, using the partitioned matrix inverse formula, we obtain our bounds, equation (8). This approach is simpler for finding out if a given price lies inside the bounds.

\subsection{Adding the positive discount factor constraint}

We can obtain sharper option price bounds by adding the constraint that the discount factor $m$ is strictly positive. This constraint rules out arbitrage portfolios with strictly positive payoffs and zero or negative prices. Such portfolios may have poor mean return/standard deviation ratios, even though they are very "good deals."

The problem for the lower bound is now ${ }^{6}$

$$
\underline{C}=\min _{\{m\}} E\left[m x^{c}\right] \text { s.t. } \mathbf{p}=E(m \mathbf{x}), E\left(m^{2}\right) \leq A^{2}, m \geq 0
$$

\footnotetext{
${ }^{6}$ Again, a discount factor respecting the constraints only exists if one chooses sufficiently large $A$, $A^{2}>\min _{m} E\left(m^{2}\right)$ s.t. $E(m \mathbf{x})=\mathbf{q}, m \geq 0$.
} 
Introducing Lagrange multipliers, the problem is

$$
\underline{C}=\min _{\{m \geq 0\}} \max _{\{\lambda, \delta\}} E\left[m x^{c}\right]+\lambda^{\prime}[E(m \mathbf{x})-\mathbf{p}]+\frac{\delta}{2}\left[E\left(m^{2}\right)-A^{2}\right]
$$

with complementary slackness (Kuhn-Tucker) condition

$$
\begin{aligned}
& \delta>0 \text { for } E\left(m^{2}\right)=A^{2} \\
& \delta=0 \text { for } E\left(m^{2}\right)<A^{2} .
\end{aligned}
$$

As suggested by Hansen, Heaton and Luttmer (1995), it is most convenient to study the conjugate (dual) optimization problem. Interchanging min and max,

$$
\underline{C}=\max _{\{\lambda, \delta\}} \min _{\{m \geq 0\}} E\left[m x^{c}\right]+\lambda^{\prime}[E(m \mathbf{x})-\mathbf{p}]+\frac{\delta}{2}\left[E\left(m^{2}\right)-A^{2}\right]
$$

If the variance constraint binds, so $\delta>0$, the inner minimization problem gives a discount factor that is a truncated linear combination of the payoffs, or an option on a linear combination of the payoffs with zero strike price,

$$
m=\max \left(-\frac{x^{c}+\lambda^{\prime} \mathbf{x}}{\delta}, 0\right)=\left[-\frac{x^{c}+\lambda^{\prime} \mathbf{x}}{\delta}\right]^{+} .
$$

The last equality defines the []$^{+}$notation for truncation. (One can derive this result in a finite-state setting by taking derivatives with respect to $m$ in each state.) Plugging equation (15) into the outer maximization of (14) and simplifying ${ }^{7}$, we obtain

$$
\underline{C}=\max _{\{\lambda, \delta>0\}} E\left\{-\frac{\delta}{2}\left[-\frac{x^{c}+\lambda^{\prime} \mathbf{x}}{\delta}\right]^{+2}\right\}-\lambda^{\prime} \mathbf{p}-\frac{\delta}{2} A^{2}
$$

We can search numerically over $\lambda, \delta$ to find the bounds, starting from the values given by proposition (4). The upper bound is found by replacing max with min and replacing $\delta>0$ with $\delta<0$.

$$
\begin{aligned}
& { }^{7} \text { The algebra. From (14), } \\
& \begin{array}{c}
C=\max _{\lambda, \delta} E\left[\left[-\frac{x^{c}+\lambda^{\prime} \mathbf{x}}{\delta}\right]^{+} x^{c}\right]+\lambda^{\prime}\left[E\left(\left[-\frac{x^{c}+\lambda^{\prime} \mathbf{x}}{\delta}\right]^{+} \mathbf{x}\right)-\mathbf{p}\right]+\frac{\delta}{2}\left[E\left(\left[-\frac{x^{c}+\lambda^{\prime} \mathbf{x}}{\delta}\right]^{+2}\right)-A^{2}\right] \\
\underline{C}=\max _{\lambda, \delta} E\left(\left[-\frac{x^{c}+\lambda^{\prime} \mathbf{x}}{\delta}\right]^{+}\left(x^{c}+\lambda^{\prime} \mathbf{x}\right)\right)+E\left(\frac{\delta}{2}\left[-\frac{x^{c}+\lambda^{\prime} \mathbf{x}}{\delta}\right]^{+2}\right)-\lambda^{\prime} \mathbf{p}-\frac{\delta}{2} A^{2} \\
\underline{C}=\max _{\lambda, \delta} E\left(-\delta\left[-\frac{x^{c}+\lambda^{\prime} \mathbf{x}}{\delta}\right]^{+2}\right)+E\left(\frac{\delta}{2}\left[-\frac{x^{c}+\lambda^{\prime} \mathbf{x}}{\delta}\right]^{+2}\right)-\lambda^{\prime} \mathbf{p}-\frac{\delta}{2} A^{2}
\end{array}
\end{aligned}
$$


What if the volatility constraint does not bind? We try each possible configuration to see which constraints bind, as follows. 1) Assume the positivity constraint is slack. Calculate the price bound without the positivity constraint, using proposition 4 . If the resulting discount factor $\underline{m}$ is nonnegative, the positivity constraint is in fact slack and this is the solution. If the discount factor $\underline{m}$ is negative in some states of nature, proceed to the next step. 2) Assume the volatility constraint is slack. Calculate the arbitrage bounds - the solutions to problem 13 without the volatility constraint. Find the minimum variance discount factor that generates the arbitrage bound. If this discount factor satisfies the volatility constraint, then the volatility constraint is in fact slack. The arbitrage bound is the solution to the problem. If not, proceed to the next step. 3) Both constraints are binding. Solve the problem (16).

Of course, it helps to be clever. One can often exploit the details of any given situation to deduce ranges in which one or the other constraints bind without explicitly going through the whole procedure. For example, we are able to show that the volatility constraint binds through the entire upper bound for a European call option, and that there is a critical value of the stock price below which it becomes slack for the lower bound.

To find the minimum variance (second moment) discount factor that generates the arbitrage bound, one can use a similar approach, as suggested by Hansen, Heaton and Luttmer (1995). Denote the lower arbitrage bound by $C_{l}$. Then, the problem is

$$
E\left(m^{2}\right)_{\min }=\min _{\{m>0\}} E\left(m^{2}\right) \text { s.t }\left[\begin{array}{c}
\mathbf{p} \\
C_{l}
\end{array}\right]=E\left(m\left[\begin{array}{c}
\mathbf{x} \\
x^{c}
\end{array}\right]\right) .
$$

Using the same dual method, this problem is equivalent to ${ }^{8}$

$$
E\left(m^{2}\right)_{\min }=\max _{\{\mathbf{v}, \mu\}}-E\left\{\left[-\left(\mu x^{c}+\mathbf{v}^{\prime} \mathbf{x}\right)\right]^{+2}\right\}-2 \mathbf{v}^{\prime} \mathbf{p}-2 \mu C_{l}
$$

Again, we search numerically for $\mathbf{v}, \mu$ to solve this problem.

\footnotetext{
${ }^{8}$ Introducing Lagrange multipliers, and interchanging $\min$ and $\max$ as above,

$$
\max _{\mathbf{v}, \mu} \min _{m \geq 0} E\left(m^{2}\right)+2 \mathbf{v}^{\prime}[E(m \mathbf{x})-\mathbf{p}]+2 \mu\left[E\left[m x^{c}\right]-C_{l}\right]
$$
}

The solution to the inner minimization is

$$
m=\left[-\left(\mu x^{c}+\mathbf{v}^{\prime} \mathbf{x}\right)\right]^{+} .
$$

Plugging this expression in the outer maximization yields the dual problem. 
Why pursue this dual approach? The first order conditions to the original problem (13) yield the same characterization of the discount factor $m$ as given by equation (15). However, in order to solve problem (13) directly, we would have to find $\lambda, \delta$ by solving the nonlinear (in $\lambda, \delta)$ constraint equations $\mathbf{p}=E(m \mathbf{x})$ and $E\left(m^{2}\right) \leq A^{2}$. Minimizations are easier numerically than nonlinear simultaneous equations solutions. (The first order conditions to the dual (16) are the constraint equations of the primal problem.) One could also search directly for a discount factor $m$, without considering any first-order conditions, but $m$ is a random variable that lies in a potentially infinite-dimensional space. $\lambda$ and $\delta$ are finitedimensional vectors.

\subsection{Application to the Black-Scholes setup}

We calculate bounds on call option prices in the Black-Scholes setup with no intermediate trading. This is an obvious example in which to try out our discrete-time methodology. We can also examine the size and economic implications of our price bounds through some examples in this well-understood setup.

The call option payoff is

$$
x^{c}=\max \left(S_{T}-K, 0\right)
$$

where $S_{T}=$ stock price at expiration, $K=$ strike price. The hedge assets are the underlying stock with current price $S$ and a riskfree bond with return $R^{f}$. We consider a larger set of hedge assets below. The stock return $R=S_{T} / S$ is lognormally distributed.

\subsubsection{Calculating moments from a lognormal model}

We need second moments for our bounds, equations (8) and (16). The stock return $R$ is lognormally distributed, with moments

$$
\begin{gathered}
E(\ln R)=\left(\mu-\frac{1}{2} \sigma^{2}\right) T ; \sigma(\ln R)=\sigma \sqrt{T} \\
E(R)=e^{\mu T} ; E\left(R^{2}\right)=e^{\left(2 \mu+\sigma^{2}\right) T}
\end{gathered}
$$

To calculate the option payoff moments, we integrate using the lognormal density. For example

$$
E\left(x^{c}\right)=S \int_{K / S}^{\infty}\left(R-\frac{K}{S}\right) f(R) d R
$$


The results are most easily summarized in a second moment matrix with $\mathbf{x}=\left[\begin{array}{ll}R & R^{f}\end{array}\right]^{\prime}$,

$$
E\left[\begin{array}{cc}
x^{c 2} & x^{c} \mathbf{x}^{\prime} \\
x^{c} \mathbf{x} & \mathbf{x} \mathbf{x}^{\prime}
\end{array}\right]=\left[\begin{array}{ccc}
S^{2} \alpha_{2}-2 K S \alpha_{1}+K^{2} \alpha_{0} & \cdot & \cdot \\
S \alpha_{2}-K \alpha_{1} & e^{\left(2 \mu+\sigma^{2}\right) T} & \cdot \\
e^{r T}\left(S \alpha_{1}-K \alpha_{0}\right) & e^{(r+\mu) T} & e^{2 r T}
\end{array}\right]
$$

where

$$
\begin{gathered}
\alpha_{0}=\int_{K / S}^{\infty} f(R) d R=N\left(\frac{\ln (K / S)-\mu T}{\sigma \sqrt{T}}+\frac{1}{2} \sigma \sqrt{T}\right) \\
\alpha_{1}=\int_{K / S}^{\infty} R f(R) d R=e^{\mu T} N\left(\frac{\ln (K / S)-\mu T}{\sigma \sqrt{T}}-\frac{1}{2} \sigma \sqrt{T}\right) \\
\alpha_{2}=\int_{K / S}^{\infty} R^{2} f(R) d R=e^{\left(2 \mu+\sigma^{2}\right) T} N\left(\frac{\ln (K / S)-\mu T}{\sigma \sqrt{T}}-\frac{3}{2} \sigma \sqrt{T}\right)
\end{gathered}
$$

and $N(\cdot)=1-\Phi(\cdot)$ is the right tail of the normal density. The right hand side of these expressions follow from standard properties of log-normal distributions with some tedious algebra.

To calculate moments for the positivity case, equation (16), we again evaluate integrals against the lognormal density for the stock return. For example,

$$
E\left\{\left[-\frac{x^{c}+\lambda^{\prime} \mathbf{x}}{\delta}\right]^{+2}\right\}=\int_{0}^{\infty} \max \left[-\frac{S \max \left(R-\frac{K}{S}, 0\right)+\lambda_{0} R^{f}+\lambda_{1} R}{\delta}, 0\right]^{2} f(R) d R
$$

These integrals can also be evaluated in closed form, but the derivation and expression are so tedious and unenlightening that we relegate them to the Appendix.

\subsubsection{Results}

Figure 2 presents price bounds for a call option with one year and no trading until expiration. Table 2 presents values of the model parameters, calibrated to an index option. The discount factor volatility bound or target Sharpe ratio in the Figure is 2 times the Sharpe ratio of the bond and stock index, or $h=1.0$. The figure includes the Black-Scholes option price and the lower arbitrage bound, for reference. The upper arbitrage bound $C<S$ does not fit on the figure.

Our price bounds are much tighter than the arbitrage bounds. The bounds are naturally tighter still as the time to expiration declines. We picked the rather long one year to expiration so that the lines would show up well on the graph. Naturally, the price bounds are larger for higher values of the target Sharpe ratio. 
Table 2: Parameters used for Black-Scholes setup with infrequent trading.

Assumed parameters

\begin{tabular}{cccc}
$\mu$ & $\sigma$ & $r$ & $\mathrm{~T}$ \\
\hline 0.1222 & 0.1409 & 0.0488 & 1 year \\
\multicolumn{4}{c}{ Derived parameters } \\
$E(R)$ & $\sigma(R)$ & $R^{f}$ & {$\left[E(R)-R^{f}\right] / \sigma(R)$} \\
\hline 1.13 & 0.16 & 1.05 & 0.5
\end{tabular}

Parameters for Black-Sholes call option calcluations. The stock return $R$ is lognormally distributed, with $E(\ln R)=\left(\mu-\sigma^{2} / 2\right) T ; \sigma(\ln R)=\sigma \sqrt{T} . T$ is the time to expiration, $r$ is the continuously compounded riskfree rate. $R, R^{f}$ are gross returns.

The difference between our bounds and the Black-Scholes price - about $\$ 1$ - is a much larger fraction of the call option price for out of the money options on the left hand side of the graph. When the Black-Scholes price is $25 \not$, a $\$ 1$ difference is quite large! Figure 3 illustrates this point by plotting the implied volatilities corresponding to the upper and lower bounds rather than the bounds themselves. The upper and lower implied volatility bounds spread dramatically for out of the money options. We expect this behavior since the option payoff is less and less similar to the stock+bond portfolio payoffs as the option moves out of the money- $E\left(w^{2}\right)$ increases and $R^{2}$ decreases. Hence, we learn less about the option price from stock and bond portfolio prices. Out-of-the-money options are harder to hedge with the underlying asset, suggesting that the Black-Scholes formula is more sensitive to infrequent trading for such options.

Figures 2 and 3 present price bounds with and without the positive discount factor restriction. The upper bounds are identical to the accuracy of the graph. The lower bound without positivity can and does fall below the lower arbitrage bound of zero for out of the money options. The lower bound with positivity becomes the arbitrage bound below a price of about $\$ 85$; in these cases the volatility bound on the discount factor is slack.

There is an interesting region labeled " $\mathrm{A}$ " in which the lower bound with volatility and positivity constraints (solid line with solid symbols) lies inside the intersection of the arbitrage bound $C>0$ and the lower bound with only a volatility constraint (dashed line with open symbols). Intersecting two constraints on the discount factor yields tighter price implications than intersecting two price regions formed from each discount factor restriction 
separately. In region A, there are strictly positive discount factors that price the option, but every one of them has excessive volatility. There are also discount factors with low volatility that generate the option prices, but every one of these is negative in some state of nature.

One may ask, are we interested in ruling out prices in region $A$ ? The answer is yes, but the interpretation is subtle. If an option price lies outside the arbitrage bounds ( $m \geq 0$ constraint), we know that there is an arbitrage opportunity. If an option lies outside the bounds that only impose discount factor volatility, we know that there is a portfolio of stock, bond and option that yields a high Sharpe ratio. If an option price lies in region $\mathrm{A}$, we know that a nonsatiated trader whose marginal utility has less than the given variance (would buy any security with Sharpe ratio greater than $h$ ) can increase his utility level by buying the option (proposition 2): However, the strategy is not simply an arbitrage or Sharpe ratio strategy.

On the other hand, region $A$ is small in figure 2. The region is even smaller for cases frequently encountered in practice, featuring shorter horizons, options less out of the money, and more hedging assets. Furthermore, calculating bounds that only impose the discount factor volatility constraint and arbitrage bounds is often numerically easier than calculating bounds that impose both volatility and positivity constraints. Therefore, intersecting price bounds rather than constraints on the discount factor can be a useful approximation in practice.

\subsection{Price bounds with multiple options}

Though the Black-Scholes formula describes dynamic hedging of an option with the underlying stock and a bond, a given option is often hedged in practice with other traded options. Since other options - say with different strike prices-have payoffs more similar to that of the option at hand, they may provide much sharper information about its price. In fact, we know from Ross (1976) that markets are complete when trading is allowed in a continuum of options with different strikes, even with no dynamic hedging.

Mechanically, we simply include other options with observed prices in the payoff space $X$. We again use the lognormal model for the stock return to calculate the required moments. Using the integral against the lognormal density to find the cross moment between two call 
options with strikes $K_{i}$ and $K_{j}$,

$$
E\left(x_{i}^{c} x_{j}^{c}\right)=S^{2} \alpha_{2}\left(K_{\max }\right)-\left(K_{i}+K_{j}\right) S \alpha_{1}\left(K_{\max }\right)+K_{i} K_{j} \alpha_{0}\left(K_{\max }\right)
$$

where $K_{\max }=\max \left(K_{i}, K_{j}\right)$. We also use the lognormal density to analytically evaluate the objective for the bound with a positive discount factor, equation (16), but again the formulas are tedious enough to be relegated to the Appendix.

\subsubsection{Results}

Figure 4 presents a contour plot of the combinations of prices of two one year options (with different strikes) that are consistent with a given Sharpe Ratio bound and only one trading interval. (Explicitly, the calculation uses equation (12)). The contours are elongated ellipses. Given the stock price, the price of the option with strike 110 must move closely, but not perfectly with the price of a strike-100 option, or a large Sharpe ratio opportunity is created.

Figure 5 shows option price bounds as a function of strike price when trading is allowed in the underlying asset, the riskless asset, and three other options whose prices are observed and plotted as black squares. The calculation makes no assumptions about where these prices of the other options come from; specifically they need not conform to the Black-Scholes formula. We again assume one period to expiration, no trading during that period, and a lognormal distribution for the underlying asset.

The arbitrage bounds ( $m \geq 0$ constraint) for filling in across strike prices are already quite tight, at least compared to the arbitrage bounds for hedging an option with the underlying asset and a bond. In between traded options, our upper bounds are the same as the arbitrage bounds. The discount factor volatility constraint is slack in this case. However, our lower bounds are a good deal tighter than the lower arbitrage bounds, and much tighter beyond the last traded option. Our bounds are tighter at the left hand side of the graph, where options are more in the money and hence their payoff "closer" to that of the stock and bond. (Figure 5 has strike price not stock price on the horizontal axis.) Our bounds are looser on the right hand side, where options are farther from the money and hence more difficult to hedge. 


\section{Deltas}

It is interesting to know the sensitivity of an option price to state variables, known as its "delta." In part, this quantity is interesting because the change over time of an option's value is given by its sensitivity to a state variable times the change of the state variable over time. Thus, the sensitivity to state variables gives the hedge portfolio in a perfect replication setup. Here we do not expect a number for a delta, but (at best) a range. Similarly, there are no perfect hedges, so portfolio formation is guided by the usual mean-variance or utility optimization. Still, it is interesting to see what we can say about the range of option price sensitivity.

Here is the problem: suppose we observe an option price, which we write $C(S, V, K, T)$ with $S=$ basis asset prices, $V=$ additional state variables (if any), $K=$ strike price $T=$ time to expiration. We want to know, how would the price change if one of the state variables changes a bit? What is $C(S+\Delta S, V, K, T)$ ?

We cannot apply our methods directly to answer this question. We know how to calculate bounds on the prices of other securities. For example, we know how to calculate bounds on $C(S, V, K+\Delta K, T)$. But $C(S+\Delta S, V, K, T)$ is not a different security, it is the same security in a different state of the world. Therefore, we can only use our methods to answer this question in a straightforward way if we can make the same security in a different state of the world look like a different security. We can do this in special cases by exploiting homogeneity properties of option prices.

Consider the example of a call option written on an underlying security $S^{i}$ with strike price $K$. We make two assumptions.

Assumption 1: There is a market option price that is only a function of the state variables, $C\left(S^{i}, S, V, K, T\right)$.

Assumption 1 does not follow from our bound calculations. Just because the price must lie in a bound $[\underline{C}, \bar{C}]$ for given values of $\left(S^{i}, S, V, K, T\right)$ does not mean the price will take on the same value in that bound each time the state variables have that configuration. We 
assume here that there is some consistent market price of risk for the unobserved shocks that generates option prices; we just don't know what that price is.

Assumption 2: The option price is homogenous of degree one in the stock price and strike price, $S^{i}, K$ :

$$
C\left(\alpha S^{i}, S, V, \alpha K, T\right)=\alpha C\left(S^{i}, S, V, K, T\right)
$$

This assumption is a natural restriction: it basically says that the units (dollars or cents) of the underlying price are irrelevant. It holds for many explicit option pricing formulas, for example the Black Scholes formula ${ }^{9}$. One can derive assumption 2 from restrictions on the state variables' stochastic process. For example, it follows if the probability distribution of returns on the underlying asset $S_{T}^{i} / S_{0}$ is independent of the initial price level $S_{0}^{i}$. In turn, that condition occurs if the drift and diffusion coefficients of a continuous time model for the state variables depend only on cumulated returns, but not on the initial level of prices. However, to save space we do not digress into a treatment of necessary and sufficient conditions for homogeneity in a suitably wide variety of models.

With homogeneity, we can infer bounds on option prices in other states of the world from bounds on prices of other options in the same state of the world. Homogeneity of degree one implies

$$
\frac{\partial C}{\partial S^{i}} S^{i}+\frac{\partial C}{\partial K} K=C
$$

Hence,

$$
\frac{\partial C}{\partial S^{i}}=\frac{C}{S^{i}}-\frac{\partial C}{\partial K} \frac{K}{S^{i}}
$$

To be specific, consider our bounds on option prices as a function of strike price as in Figure 5. At a point at which we observe an option price (a black square), the upper and lower bounds collapse to the observed price, $\bar{C}=\underline{C}=C$. Thus, the maximum and minimum values of $\partial C / \partial S^{i}$ at any such point is determined by the slope of the upper and lower bounds $\partial \underline{C} / \partial K$ and $\partial \bar{C} / \partial K$ through the point $C$.

$$
\begin{aligned}
& { }^{9} \text { The Black-Scholes formula can be written } \\
& \qquad \frac{C}{S}=N\left(d_{1}\right)-\frac{K}{S} N\left(d_{2}\right) \\
& d_{1}=\frac{\ln (S / K)+\left(r+\sigma^{2} / 2\right) T}{\sigma \sqrt{T}} ; d_{2}=d_{1}-\sigma \sqrt{T}
\end{aligned}
$$

from which homogeneity' of degree one in $S, K$ is clear. 
Figure 6 presents a graph of upper and lower bounds on deltas computed in this way, using the same setup as Figure 5. For the middle option, our delta bounds coincide with the arbitrage-based delta bounds. Locally around this option strike price, the positivity constraint binds and the volatility constraint is slack; in Figure 5 upper and lower bounds leave this point with the same slope. This fact suggests that the Black-Scholes delta calculation is quite sensitive to trading assumptions.

For the left-hand, or in-the-money option, our lower delta bound is the same as the arbitrage bound in Figure 6. The arbitrage price bound and good-deal price bound give the same value for the shallower slope in Figure 5. The arbitrage bound makes no restriction on the steeper slope in Figure 5 however, resulting in an infinite delta bound. Our delta bound is much smaller.

For the right hand, or out-of-the-money option, the steeper slope of the good deal bound is the same as that of the arbitrage bound in Figure 5, so we do not improve on the arbitrage bound delta in Figure 6. However, the shallower slope is zero for the arbitrage bound, and the good deal bound improves on this, resulting in a tighter bound on delta.

In summary, we find in this illustrative calculation that good-deal delta bounds do not seem to improve on arbitrage delta bounds for intermediate options. This fact suggests that we in fact know little more than the arbitrage bounds about such deltas. Good-deal delta bounds do restrict the deltas of options with extreme strikes. 


\section{Price bounds with multiple trading periods - a re- cursive solution}

Allowing multiple trading periods and intermediate payoffs is an obvious and important extension. In the case of option pricing (and application of option pricing techniques to other random payoffs in economics and finance), we at least want to allow imperfect dynamic hedging. If we could only handle buy-and-hold situations, our technique would be of limited interest.

Here, we state and find a recursive solution to the multi-period problem. This recursive solution makes our procedure computationally feasible. Below, we extend the recursive solution by shrinking the trading interval to zero, allowing us to handle continuous time and continuous trading models in which extra state variables rather than discrete trading are the source of market incompleteness.

Consider the same problem as above, pricing an option with payoff $x^{c}$ with basis assets whose payoffs and prices are observed, and whose distribution we can model. Now we allow one hedge portfolio rebalancing half-way to expiration. Using time periods 0 for today, and 1 and 2 for the rebalancing date and payoff date respectively, the objective function for the lower price bound is

$$
\underline{C}_{0}=\min _{\left\{m_{1}>0, m_{2}>0\right\}} E_{0}\left(m_{1} m_{2} x^{c}\right)
$$

where $E_{t}(\cdot)=E\left(\cdot \mid I_{t}\right)$ denotes conditional expectation.

Next, the constraints. The discount factors $m_{1}$ and $m_{2}$ must price the basis assets, so we require

$$
\begin{gathered}
E_{0}\left(m_{1} \mathbf{p}_{1}\right)=\mathbf{p}_{0} \\
E_{1}\left(m_{2} \mathbf{x}_{2}\right)=\mathbf{p}_{1} \text { for all information sets } I_{1},
\end{gathered}
$$

where $\mathbf{p}_{i}$ denotes the vector of prices of the basis assets at time $i$ and $\mathbf{x}_{2}$ the final payoff of the assets at time 2 . We generalize the discount factor volatility constraint to the requirement 
that the volatility in each period respect a bound ${ }^{10}$,

$$
E_{0}\left(m_{1}^{2}\right) \leq A_{0}^{2} ; E_{1}\left(m_{2}^{2}\right) \leq A_{1}^{2}
$$

Now we can state the two period problem and its recursive solution.

Proposition 5 The two-period problem

$$
\begin{gathered}
\underline{C}_{0}=\min _{\left\{m_{1}, m_{2}\right\}} E_{0}\left(m_{1} m_{2} x^{c}\right) \text { s.t. } \\
\mathbf{p}_{0}=E_{0}\left(m_{1} \mathbf{p}_{1}\right), E_{0}\left(m_{1}^{2}\right) \leq A_{0}^{2}, m_{1} \geq 0, \\
\mathbf{p}_{1}=E_{1}\left(m_{2} \mathbf{x}_{2}\right), E_{1}\left(m_{2}^{2}\right) \leq A_{1}^{2} \quad \forall I_{1}, m_{2} \geq 0
\end{gathered}
$$

has the same solution as the sequence of one-period problems

$$
\begin{gathered}
\underline{C}_{1}=\min _{\left\{m_{2}\right\}} E_{1}\left(m_{2} x^{c}\right) \text { s.t. } \mathbf{p}_{1}=E_{1}\left(m_{2} \mathbf{x}_{2}\right), E_{1}\left(m_{2}^{2}\right) \leq A_{1}^{2}, m_{2} \geq 0 \\
\underline{C}_{0}=\min _{\left\{m_{1}\right\}} E_{0}\left(m_{1} \underline{C}_{1}\right) \text { s.t. } \mathbf{p}_{0}=E_{0}\left(m_{1} \mathbf{p}_{1}\right), E_{0}\left(m_{1}^{2}\right) \leq A_{0}^{2}, m_{1} \geq 0 .
\end{gathered}
$$

Proof: Since the constraints are independent of the choice variables, we can interchange the $\min$ and the expectation ${ }^{11}$, and write the problem as

$$
\underline{C}_{0}=\min _{\left\{m_{1}\right\}} E_{0}\left\{m_{1}\left[\min _{\left\{m_{2}\right\}} E_{1}\left(m_{2} x^{c}\right) \text { s.t. } \mathbf{p}_{1}=E_{1}\left(m_{2} \mathbf{x}_{2}\right), E_{1}\left(m_{2}^{2}\right) \leq A_{1}^{2}, m_{2} \geq 0\right]\right\} \text { s.t. }
$$

${ }^{10}$ An alternative constraint might be that the two-period Sharpe ratio is below a given value,

$$
E_{0}\left[\left(m_{1} m_{2}\right)^{2}\right] \leq A^{2}
$$

Then this problem is just like the preceding one, with an additional trading opportunity. Alas, this constraint does not lead to a recursive solution. Of course, constraint (18) implies a constraint of the form (17),

$$
E_{0}\left[\left(m_{1} m_{2}\right)^{2}\right]=E_{0}\left[m_{1}^{2} E_{1}\left(m_{2}^{2}\right)\right] \leq E_{0}\left[m_{1}^{2} A_{1}^{2}\right] \leq A_{0}^{2} A_{1}^{2}
$$

But the converse is not true.

The Sharpe ratio scales with the time period. An appropriate numerical value to impose for a six-month trading interval is the square root of the appropriate value to impose for a one-year trading interval, i.e. $A_{0}^{2}=A_{1}^{2}=\sqrt{A^{2}}$.

${ }^{11}$ Readers who are justly suspicious of interchanging the min and expectation operators, and who find the appeal to Bellman's principle unconvincing, may wish to examine the following more detailed argument. Write the two period problem without positivity in a finite-state environment as

$$
\begin{gathered}
\underline{C}_{0}=\min _{m_{1}, m_{2}} \sum_{i} \pi(i) m_{1}(i) \sum_{j} \pi(j \mid i) m_{2}(i, j) x_{c}(i, j) \text { s.t. } \\
\sum_{i} \pi(i) m_{1}(i) \mathbf{x}(i)=\mathbf{p}_{0} ; \sum_{j} \pi(j \mid i) m_{2}(i, j) \mathbf{x}(i, j)=\mathbf{p}_{1}(i) \forall i
\end{gathered}
$$




$$
\mathbf{p}_{0}=E_{0}\left(m_{1} \mathbf{p}_{1}\right), E_{0}\left(m_{1}^{2}\right) \leq A_{0}^{2}, m_{1} \geq 0
$$

Of course, the inner minimization is the one-period bound solved above. Thus, we have the recursive statement.

This proposition is just an application of Bellman's Principle of Optimality (see e.g. Bertsekas and Shreve 1978). The original problem is a stochastic control problem with a state process that is independent of the control $m_{t}$.

The recursive statement extends in an obvious way to multiple periods. The $A$ constraints may be functions of information (state), and thus allow desired risk premia that vary over time or with state. Finally, it is easy to extended this setup to include basis assets and derivatives with intermediate payoffs.

\subsection{A multiperiod multinomial application}

Here we extend our discrete-trading Black-Scholes world to multiple trading periods. We know that as the number of trading periods increases, our upper and lower bounds should collapse to the Black-Scholes price. Quantitatively, it is interesting to know how quickly this occurs.

We need to solve backwards for each trading date a problem similar to the one we solved above for the one period case. Now $x^{c}$ represents the value next period of the lower (upper)

$$
\sum_{i} \pi(i) m_{1}(i)^{2} \leq A_{0}^{2} ; \sum_{j} \pi(j \mid i) m_{2}(i, j)^{2} \leq A_{1}^{2}(i) \forall i
$$

Introduce Lagrange multipliers $\lambda_{0}, \pi(i) \lambda_{1}(i), \delta_{0} / 2$, and $\pi(i) \delta_{1}(i) / 2$ on the constraints, and take derivatives with respect to $m_{2}(i, j)$,

$$
\partial / \partial m_{2}(i, j): m_{2}(i, j)=-\frac{m_{1}(i) x_{c}(i, j)+\lambda_{1}(i)^{\prime} \mathbf{x}(i, j)}{\delta_{1}(i)}
$$

$m_{1}(i)$ is just a number in the $I_{1}$ information set, so this $m_{2}$ is of the same form as in the one period problem equation (15), and the time 1 pricing constraint and Sharpe ratio constraint then give the same solution as the one-period problem. Taking derivatives with respect to $m_{1}$,

$$
\begin{gathered}
\partial / \partial m_{1}(i): m_{1}(i)=-\frac{\lambda_{0}^{\prime} \mathbf{x}(i)+\sum_{j} \pi(j \mid i) m_{2}(i, j) x_{c}(i, j)}{\delta_{0}} \\
m_{1}(i)=-\frac{\lambda_{0}^{\prime} \mathbf{x}(i)+\underline{C}(i)}{\delta_{0}}
\end{gathered}
$$

This is a discount factor of the same form, with the first-period lower bound in the place of the option payoff. 
price bound for the option (see equation 19) for all the trading dates but the last one. Thus, at each trading date we need models for the second moments of the bounds at the next trading date. Conceptually, this is not difficult: we have assumed a lognormal distribution for the underlying asset, and we have an analytical formula for the bounds at the last trading date. However, the price bounds one period before expiration are highly non-linear functions of the price of the underlying security. Integrating to compute these moments is ugly, and the problem gets uglier each time we move back one trading date.

Instead, we approximate the lognormal distribution of stock returns by a discrete multinomial distribution. Many option pricing problems are most conveniently solved by making a multinomial approximation, and we also use this example to show how to calculate good-deal bounds in a multinomial environment.

We discretize the possible values of the underlying asset price to $N$ possible values, $S_{1}, S_{2} \ldots S_{N}$. We use the lognormal distribution to assign transition probabilities between the $N$ states,

$$
\pi_{i \rightarrow j}=\pi\left(S_{t+\Delta t}=S_{j} \mid S_{t}=S_{i}\right)
$$

We use a binomial distribution with interval 1 day to generate the probabilities and number of states, e.g. 30 states for a monthly trading interval. (Not $2^{30}$, since the tree recombines.) We do this here to make sure our results are consistent across choices for the trading interval. In making calculations for a fixed trading interval, one could simply approximate the distribution, e.g. $\pi_{j}=f\left(S_{j}\right) \Delta S$.

Denote the payoffs on the basis assets from one state to the next by

$$
\mathbf{p}_{i}=\left[\begin{array}{c}
1 \\
1
\end{array}\right] ; \quad \mathbf{x}_{i \rightarrow j}=\left[\begin{array}{c}
S_{j} / S_{i} \\
e^{r \Delta t}
\end{array}\right]
$$

The analytic solution to our problem without positivity, equation (8) is, using state $i$ time $t$ notation,

$$
\begin{gathered}
C_{i, t}=\mathbf{p}_{i}^{\prime} E_{i}\left(\mathbf{x} \mathbf{x}^{\prime}\right)^{-1} E_{i}\left(\mathbf{x} \underline{C}_{t+\Delta t}\right)-\sqrt{A^{2}-\mathbf{p}_{i}^{\prime} E_{i}\left(\mathbf{x} \mathbf{x}^{\prime}\right)^{-1} \mathbf{p}_{i}} \sqrt{E_{i}\left(w^{2}\right)} \\
E_{i}\left(w^{2}\right)=E_{i}\left(\underline{C}_{t+\Delta t}^{2}\right)-E_{i}\left(\underline{C}_{t+\Delta t} \mathbf{x}^{\prime}\right) E_{i}\left(\mathbf{x} \mathbf{x}^{\prime}\right)^{-1} E_{i}\left(\mathbf{x} \underline{C}_{t+\Delta t}\right)
\end{gathered}
$$

We use our discrete model to calculate the moments on the right hand side in each state $i$,

$$
E_{i}\left(\mathbf{x} \mathbf{x}^{\prime}\right)=\sum_{j} \pi_{i \rightarrow j} \mathbf{x}_{i \rightarrow j} \mathbf{x}_{i \rightarrow j}^{\prime}
$$




$$
\begin{gathered}
E_{i}\left(\mathbf{x} \underline{C}_{t+\Delta t}\right)=\sum_{j} \pi_{i \rightarrow j} \mathbf{x}_{i \rightarrow j} \underline{C}_{j, t+\Delta t} \\
E_{i}\left(\underline{C}_{t+\Delta t}^{2}\right)=\sum_{j} \pi_{i \rightarrow j} \underline{C}_{j, t+\Delta t}^{2}
\end{gathered}
$$

As outlined above, we first try this analytic solution. The resulting $m$ is a vector of values for each state, we check that each element is nonnegative. If not, we proceed as outlined above to check for an arbitrage bound ${ }^{12}$ solution with a slack volatility constraint and finally search for a solution to the case with both constraints binding as specified by equation (16).

\subsubsection{Results}

Figure 7 presents upper and lower bounds for an at-the money call option as we increase the number of trading dates. The option and parameters are all the same as in the examples studied above. The upper and lower price bounds fall quickly until we reach a two week trading interval (21 dates). Further reductions in the option price bounds require much more frequent trading.

\footnotetext{
${ }^{12}$ Ritchken (1985) derives arbitrage bounds for this model.
} 


\section{Continuous time and missing assets}

An important class of incomplete market problems results when assets are missing, rather than as a result of discrete trading opportunities. For example, we may not observe or infer the risk prices of nontraded state variables such as the interest rate or stochastic volatility. These missing markets rule out arbitrage pricing, even with continuous trading. We develop and apply price bounds for such situations.

\subsection{Discount factors in continuous time}

Let $S_{t}$ denote a price process, i.e. the cumulative value of some asset or portfolio. A discount factor is a positive process $\Lambda_{t}$ such that the product $\Lambda_{t} S_{t}$ is a martingale, i.e.

$$
\Lambda_{t} S_{t}=E_{t}\left(\Lambda_{t+T} S_{t+T}\right)
$$

For an investor with a time-additive and state independent utility function, the marginal utility of consumption or wealth is a discount factor,

$$
\Lambda_{t}=\delta^{t} u^{\prime}\left(c_{t}\right)
$$

Again, we suppress time subscripts where they are not necessary, e.g. we write $\Lambda=\Lambda_{t}$.

We recover the continuous time equivalent to $1=E(m R)$ by taking the limit $T \rightarrow 0$ of the martingale condition (21),

$$
0=\frac{E_{t}(d \Lambda S)}{\Lambda S}=\frac{E_{t}(d \Lambda)}{\Lambda}+\frac{E_{t}(d S)}{S}+\frac{E_{t}(d \Lambda d S)}{\Lambda S} .
$$

If the assets $S$ pay dividends at a rate $D d t$ then (22) generalizes naturally to

$$
0=\frac{E_{t}(d \Lambda S)}{\Lambda S}+\frac{D}{S} d t
$$

As in discrete time, we can derive restrictions on discount factors so that they price assets. For example, if there is a money market fund with value $B_{t}$ that pays the instantaneous interest rate $r$,

$$
\frac{d B}{B}=r_{t} d t
$$


then equation (22) implies

$$
E_{t}(d \Lambda)=-r_{t} \Lambda d t
$$

This equation is the continuous-time counterpart to $R^{f}=1 / E(m)$.

As discrete time pricing is often expressed in terms of expected returns, continuous time pricing is often expressed by "market prices of risk." If a risky asset follows

$$
\frac{d S}{S}=\mu_{S} d t+\sigma_{S} d z
$$

and the interest rate is $r$, then equation (23) implies

$$
E_{t} \frac{d S}{S}+\left(\frac{D}{S}-r\right) d t=-E_{t} \frac{d \Lambda d S}{\Lambda S}
$$

This equation is the continuous-time counterpart to the expression $E\left(R^{e}\right)=-\operatorname{cov}\left(m, R^{e}\right) / E(m)$ for excess returns $R^{e}$. Thus, a security with diffusion $d z$ should have an expected excess return equal to

$$
-E_{t}\left(\frac{d \Lambda}{\Lambda} d z\right)
$$

This quantity (sometimes divided by an instantaneous variance or standard deviation) is the "market price" of the risk $d z$.

The analog to proposition 1 comes from plugging the model (24) into the first order condition (22).

$$
0=-r d t+\mu_{S} d t+\frac{E_{t}(d \Lambda d S)}{\Lambda S} \leq\left(\mu_{S}-r\right) d t+\frac{\sqrt{E_{t}\left(d \Lambda^{2}\right)}}{\Lambda} \sigma_{S} \sqrt{d t}
$$

Hence, the maximum Sharpe ratio of any security priced by $\Lambda$ is governed by the volatility of $\Lambda$

$$
\frac{\left(\mu_{S}-r\right)^{2}}{\sigma_{S}^{2}} d t \leq \frac{E_{t} d \Lambda^{2}}{\Lambda^{2}}
$$

This equation is the continuous time counterpart to $\left|E\left(R^{e}\right)\right| / \sigma\left(R^{e}\right) \leq \sigma(m) / E(m)$. 


\subsection{Deriving price bounds}

We model the price processes of an $n_{S^{-}}$-dimensional vector of basis assets by ${ }^{13}$

$$
\frac{d S}{S}=\mu_{S}(S, V, t) d t+\sigma_{S}(S, V, t) d z
$$

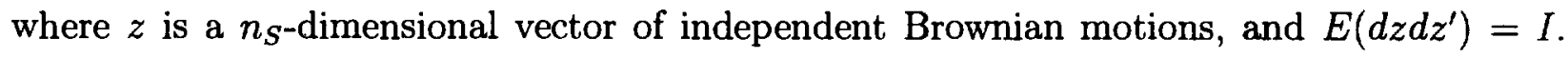
The basis assets may pay dividends at rate $D(S, V, t) d t$. Let $V$ represent an $n_{V}$-dimensional vector of state variables, for example stochastic volatility, that follow

$$
d V=\mu_{V}(S, V, t) d t+\sigma_{V z}(S, V, t) d z+\sigma_{V w}(S, V, t) d w
$$

where $d w$ is an $n_{V}$-dimensional vector of Brownian increments orthogonal to $d z: E\left(d w d w^{\prime}\right)=$ $I ; E\left(d w d z^{\prime}\right)=0$.

Other diffusion models can be expressed in the form (26) - (27) without loss of generality. A model with more shocks than variables can always be expressed as a model with the same number of shocks as variables, by constructing linear combinations of the original shocks. A model with non-orthogonalized shocks can be expressed in the form (26) - (27) by suitably orthogonalizing the shocks ${ }^{14}$.

\footnotetext{
${ }^{13}$ Rather than complicate the notation, understand division to operate element by element on vectors, e.g., $d S / S=\left[\begin{array}{lll}d S_{1} / S_{1} & d S_{2} / S_{2} & \cdots\end{array}\right]$. When explicit enumeration of arguments is not necessary, we write $S$ for $S(t)$ and $\mu_{S}$ and $\sigma_{S}$ for $\mu_{S}(S, V, t)$ and $\sigma_{S}(S, V, t)$. We assume that all diffusion paramaters, $\mu_{S}(S, V, t)$, $\sigma_{S}(S, V, t), \mu_{V}(S, V, t), \sigma_{V}(S, V, t)$, etc. are continuous in all their arguments. We assume that all variancecovariance matrices such as $\sigma_{S}(S, V, t) \sigma_{S}(S, V, t)^{\prime}, \sigma_{V}(S, V, t) \sigma_{V}(S, V, t)^{\prime}$ are non-singular for all $S \in \mathcal{R}^{n_{S}}$, $V \in \mathcal{R}^{n_{V}}, t \in[0, T]$.

${ }^{14}$ Suppose we start instead with

$$
\begin{gathered}
\frac{d S}{S}=\mu_{S} d t+\sigma_{S y} d y \\
d V=\mu_{V} d t+\sigma_{V y} d y
\end{gathered}
$$
}

where $d y$ is an $n_{y}=n_{S}+n_{V}-$ dimensional vector of Brownian increments. Define matrices

$$
Q_{w}=\operatorname{null}\left(\sigma_{S y}\right)^{\prime} ; Q_{z}=\operatorname{arth}\left(\sigma_{S y}\right)^{\prime}
$$

$\mathrm{Null}(\cdot)$ creates an orthogonal basis for the nullspace of $\sigma_{S y}$; it creates a $n_{V} \times n_{y}$ matrix $Q_{w}$ such that $\sigma_{S y} Q_{w}^{\prime}=0, Q_{w} Q_{w}^{\prime}=I_{V}$. Orth(·) creates an orthogonal basis for the column space of $\sigma_{S y}$; it creates an $n_{S} \times n_{y}$ matrix $Q_{z}$ such that $Q_{z} Q_{z}^{\prime}=I_{S}, \quad \sigma_{S y} Q_{z}^{\prime}=\sigma_{S y}$ and $Q_{w} Q_{z}^{\prime}=0$. Finally, since nullspace and column space add up to everything, $Q_{w}^{\prime} Q_{w}+Q_{z}^{\prime} Q_{z}=I_{y} \cdot\left(Q_{z}\right.$ factors the projection onto the column space of $\sigma_{S y}: Q_{z}^{\prime} Q_{z}=\sigma_{S y}^{\prime}\left(\sigma_{S y} \sigma_{S y}^{\prime}\right)^{-1} \sigma_{S y}$, and $Q_{w}$ similarly factors $I_{y}-\sigma_{S y}^{\prime}\left(\sigma_{S y} \sigma_{S y}^{\prime}\right)^{-1} \sigma_{S y}$. Null and Orth are the names of GAUSS commands that achieve these decompositions.) Now write

$$
\begin{gathered}
\frac{d S}{S}=\mu_{S} d t+\sigma_{S y}\left[Q_{w}^{\prime} Q_{w}+Q_{z}^{\prime} Q_{z}\right] d y=\mu_{S} d t+\sigma_{S y} Q_{z}^{\prime} Q_{z} d y \\
d V=\mu_{V} d t+\sigma_{V y} Q_{w}^{\prime} Q_{w} d y+\sigma_{V y} Q_{z}^{\prime} Q_{z} d y
\end{gathered}
$$


We assume that there is an instantaneously risk free rate, a money market security such that

$$
\frac{d B}{B}=r(S, V, t) d t
$$

This assumption is not essential, but it simplifies the algebra and presentation. When $r$ varies stochastically, it is one of the state variables in $V$.

We want to price an asset that pays continuous dividends at rate $x_{t}^{c} d t$ and a terminal payment $x_{T}^{c}$.

The risks associated with $n_{V}$ non-traded stochastic variables cannot be hedged. Therefore the markets are incomplete, at least before the introduction of derivatives. This continuoustime setup with incomplete markets is similar to He and Pearson (1992) and Karatzas et al. (1991).

The problem is now to choose a discount factor process to minimize (maximize) the asset price

$$
\underline{C}_{t}=\min _{\left\{\Lambda_{s}, t<s \leq T\right\}} E_{t} \int_{s=t}^{T} \frac{\Lambda_{s}}{\Lambda_{t}} x_{s}^{c} d s+E_{t}\left(\frac{\Lambda_{T}}{\Lambda_{t}} x_{T}^{c}\right)
$$

subject to the constraints that 1) the discount factor prices the basis assets $S, B$ at each moment in time, 2) the instantaneous volatility of the discount factor process is less than a prespecified value $A^{2}$ (or, more generally, less than a process $A(S, V, \tau)^{2}$ ) at each moment in time) and 3) the discount factor is positive $\Lambda_{s} \geq 0, t \leq s \leq T$. The upper bound follows by replacing min with max.

\subsubsection{Recursive and then differential statement of the problem}

As before, we can break up the original problem into a minimization from $t$ to $u$ and then from $u$ to $T$. The expectation and minimization can be interchanged, so the minimization from $u$ to $T$ is the lower bound at date $u$. Thus, problem (28) is equivalent to the recursive formulation

$$
C_{u}=\min _{\{\Lambda(s), u \leq s \leq T\}} E_{u} \int_{s=u}^{T} \frac{\Lambda_{s}}{\Lambda_{u}} x_{s}^{c} d s+E_{t}\left(\frac{\Lambda_{T}}{\Lambda_{u}} x_{T}^{c}\right)
$$

We recover the orthogonalized representation (26) - (27) with the definitions

$$
\begin{gathered}
d w=Q_{w} d y ; \quad d z=Q_{z} d y \\
\sigma_{S}=\sigma_{S y} Q_{z}^{\prime} ; \sigma_{V z}=\sigma_{V y} Q_{z}^{\prime} ; \quad \sigma_{V w}=\sigma_{V y} Q_{w}^{\prime}
\end{gathered}
$$




$$
\underline{C}_{t}=\min _{\{\Lambda(s), t \leq s \leq u\}} E_{t} \int_{s=t}^{u} \frac{\Lambda_{s}}{\Lambda_{t}} x_{s}^{c} d s+E_{t}\left(\frac{\Lambda_{u}}{\Lambda_{t}} C_{u}\right)
$$

subject to the constraints.

Using this recursive statement with $u=t+\Delta t$ we obtain

$$
\underline{C}_{t} \Lambda_{t}=\min _{\{\Delta \Lambda\}}\left\{x^{c} \Delta t+\underline{C}_{t+\Delta t}\left(\Lambda_{t}+\Delta \Lambda\right)\right\} .
$$

Letting $\Delta t \rightarrow 0$, we can write our problem in differential form,

$$
0=\frac{x_{t}^{c}}{\underline{C}} d t+\min _{\{d \Lambda\}} \frac{E_{t} d(\Lambda \underline{C})}{\Lambda \underline{C}}
$$

subject to the constraints.

Condition (29) has a nice interpretation. After the minimization, the discount factor process $\underline{\Lambda}$ that generates the lower bound and the lower bound $\underline{C}$ satisfy

$$
0=\frac{x^{c}}{\underline{C}} d t+\frac{E_{t} d(\underline{\Lambda C})}{\underline{\Lambda C}} .
$$

This is the basic pricing equation (23); it is the condition that $\underline{\Lambda}$ generates the lower bound $\underline{C}$ as the price of a security that pays a dividend stream $x^{c} . \underline{\Lambda}$ is one particular extension of the discount factors that price the basis assets. $\bar{\Lambda}$, produced by max instead of $\min$ in equation (29), is another particular extension, which prices the basis assets and generates the upper price bound as the price of a security that pays $x^{c}$.

\subsubsection{Constraints}

Now we express the constraints that a discount factor $\underline{\Lambda}$ prices the basis assets and satisfies the volatility bound. As in the discrete time case, we orthogonalize the discount factor. Then, our minimization problem is simply to choose the weights on the orthogonal components.

Proposition $6 \Lambda_{t}$ is a discount factor driven by $d z$, dw that prices the basis assets and if and only if it can be represented as ${ }^{15}$

$$
\frac{d \Lambda}{\Lambda}=\frac{d \Lambda^{*}}{\Lambda^{*}}-v d w
$$

\footnotetext{
${ }^{15}$ We use differential notation for clarity; sticklers for continuous time notation may prefer integral forms, for example

$$
\Lambda_{t}=-\int_{0}^{t} \Lambda_{s} r_{s} d s-\int_{0}^{t} \Lambda_{s} \tilde{\mu}_{S}^{\prime} \Sigma_{S}^{-1} \sigma_{S} d z-\int_{0}^{t} \Lambda_{s} v d w \text { a.s. }
$$
}


where

$$
\begin{gathered}
\frac{d \Lambda^{*}}{\Lambda^{*}} \equiv-r d t-\tilde{\mu}_{S}^{\prime} \Sigma_{S}^{-1} \sigma_{S} d z \\
\tilde{\mu}_{S} \equiv \mu_{S}+\frac{D}{S}-r ; \quad \Sigma_{S}=\sigma_{S} \sigma_{S}^{\prime} .
\end{gathered}
$$

and $v$ is an arbitrary ${ }^{16} 1 \times n_{V}$ matrix.

Proof: Just verify that $\Lambda$ satisfies the basic pricing equation (23), for the money market and basis assets. Liberally using $E(d S d w)=0$ and $E(d z d w)=0$,

$$
\begin{gathered}
E_{t} \frac{d \Lambda}{\Lambda}=E_{t} \frac{d \Lambda^{*}}{\Lambda^{*}}-r d t \\
E_{t} \frac{d \Lambda S}{\Lambda S}+\frac{D}{S} d t=E_{t} \frac{d S}{S}+E_{t} \frac{d \Lambda^{*}}{\Lambda^{*}}+E_{t} \frac{d \Lambda^{*} d S}{\Lambda^{*} S}+\frac{D}{S} d t= \\
=\left[\mu_{S}-r-\tilde{\mu}_{S}^{\prime} \Sigma_{S}^{-1} \sigma_{S} \sigma_{S}^{\prime}+\frac{D}{S}\right] d t=0 .
\end{gathered}
$$

The converse holds by projection, as in proposition 3 .

This proposition is the obvious continuous-time counterpart to proposition 3 , and has the same geometric interpretation as in Figure 1. We can let $d \Lambda / \Lambda$ load on additional shocks, orthogonal to both $V$ and $S$. For this reason the proposition qualifies "driven by $d z, d w$ ". However, our minimization or maximization of asset prices will again put such loading to zero. (This proposition and proof are similar to Proposition 3.1. in He and Pearson 1992.)

The volatility constraint is

$$
\frac{1}{d t} E_{t} \frac{d \Lambda^{2}}{\Lambda^{2}} \leq A^{2}
$$

and hence

$$
v v^{\prime} \leq A^{2}-\frac{1}{d t} E_{t} \frac{d \Lambda^{* 2}}{\Lambda^{* 2}}=A^{2}-\tilde{\mu}_{S}^{\prime} \Sigma_{S}^{-1} \tilde{\mu}_{S}
$$

By expressing the constraints in this way, we have again reduced the problem of choosing the stochastic process for $\Lambda$ to the choice of loading $v$ on the noises $d w$ with unknown prices, subject to a quadratic constraint on $v v^{\prime}$.

\footnotetext{
${ }^{16}$ We require $E\left[\exp \left(\frac{1}{2} \int_{0}^{T}\left|\tilde{\mu}_{S}^{\prime} \Sigma_{S}^{-1} \sigma_{S}\right|^{2} d t\right)\right]<\infty$ and $E\left[\exp \left(\frac{1}{2} \int_{0}^{T}|v|^{2} d t\right)\right]<\infty$, to ensure that the stochastic integrals that describe the dynamics of $\Lambda$ are well-defined.
} 


\subsubsection{Solutions: a characterization}

We characterize the solution to our problem with three propositions. First, we characterize the discount factor and lower bound processes. We characterize the lower bound process via the coefficients $\mu_{\underline{C}}, \sigma_{\underline{C}_{z}}$ and $\sigma_{\underline{C}_{w}}$ in a representation

$$
\frac{d \underline{C}}{\underline{C}}=\mu_{\underline{C}}(S, V, t) d t+\sigma_{\underline{C}_{z}}(S, V, t) d z+\sigma_{\underline{C} w}(S, V, t) d w .
$$

Proposition 7 The lower bound discount factor $\Lambda_{t}$ follows

$$
\frac{d \underline{\Lambda}}{\underline{\Lambda}}=\frac{d \Lambda^{*}}{\Lambda^{*}}-\underline{v} d w
$$

and $\mu_{\underline{C}}, \sigma_{\underline{C}_{z}}$ and $\sigma_{\underline{C} z}$ satisfy the restriction

$$
\mu_{\underline{C}}+\frac{x^{c}}{\underline{C}}-r=-\frac{1}{d t} E_{t}\left[\frac{d \Lambda^{*}}{\Lambda^{*}} \sigma_{\underline{C} z} d z\right]+\underline{v} \sigma_{\underline{C} w}^{\prime}
$$

where

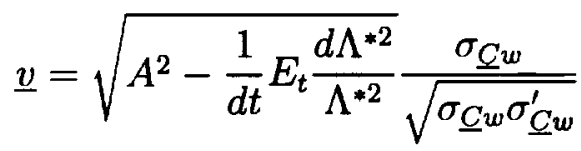

The upper bound process $\bar{C}_{t}$ and discount factor $\bar{\Lambda}_{t}$ have the same representation with $\bar{v}=-\underline{v}$.

Proof: The problem (29) can be written

$$
0=\frac{x^{c}}{\underline{C}} d t+\min _{\{d \Lambda\}} E_{t} \frac{d(\Lambda \underline{C})}{\Lambda \underline{C}}
$$

Substituting from equation (30) in order to impose the pricing constraint, the objective is

$$
0=\frac{x^{c}}{\underline{C}} d t_{t}+E_{t} \frac{d\left(\Lambda^{*} \underline{C}\right)}{\Lambda^{*} \underline{C}}-\min _{\{v\}} v E_{t} d w \frac{d \underline{C}}{\underline{C}}
$$

We write the lower bound $C$ as a diffusion process, equation (32), and we derive restrictions on the drift and diffusion functions. Substituting, we can express

$$
v E_{t} d w \frac{d \underline{C}}{\underline{C}}=v \sigma_{\underline{C} w}^{\prime} d t
$$

Hence, (35) becomes

$$
0=\frac{x^{c}}{\underline{C}}+\frac{1}{d t} E_{t} \frac{d\left(\Lambda^{*} \underline{C}\right)}{\Lambda^{*} \underline{C}}-\min _{\{v\}} v \sigma_{\underline{C} w}^{\prime} \text { s.t. } v v^{\prime} \leq A^{2}-\frac{1}{d t} E_{t} \frac{d \Lambda^{* 2}}{\Lambda^{* 2}} .
$$


This is a linear objective in $v$ with a quadratic constraint. Therefore, as long as $\sigma_{\underline{C} w} \neq 0$, the constraint binds. Introducing a Lagrange multiplier and taking the first order condition with respect to $v$, we have

$$
v=\alpha \sigma_{\underline{\underline{C}} w}
$$

The multiplier $\alpha$ is determined to satisfy the volatility constraint, yielding

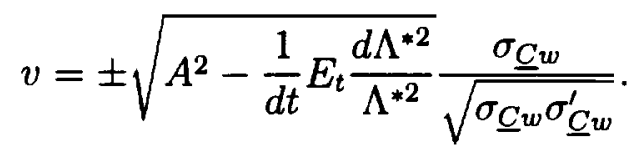

The + sign generates the minimum in (36) since

$$
\sigma_{\underline{C} w} \sigma_{\underline{C} w}^{\prime} \geq 0
$$

Similarly, a - sign generates the upper price bound. (If $\sigma_{\underline{C} w}=0$, the price bound does not load on the state variables with unobserved prices. Hence, any $v$ leads to the same price bound. In this case we can most simply take $v=0$.) Plugging the optimal value for $v$ in (36) gives

$$
0=\frac{x^{c}}{\underline{C}}+\frac{1}{d t} E_{t} \frac{d \Lambda^{*} \underline{C}}{\Lambda^{*} \underline{C}}-\underline{v} \sigma_{\underline{C} w}^{\prime} .
$$

For clarity, we write the middle term as

$$
\frac{1}{d t} E_{t} \frac{d \Lambda^{*} \underline{C}}{\Lambda^{*} \underline{C}}=-r+\mu_{\underline{C}}+E_{t} \frac{d \Lambda^{*}}{\Lambda^{*}}\left(\sigma_{\underline{C} z} d z+\sigma_{\underline{C} w} d w\right) .
$$

Since $d \Lambda^{*}$ is orthogonal to $d w$, we obtain the restriction(34).

The statement of this proposition is a straightforward analog to proposition 4 and has a similar geometric interpretation as shown in Figure 1. $d \Lambda^{*} / \Lambda^{*}$, is the combination of basis asset shocks that prices the basis assets by construction, in analogy to $x^{*}$. The term $\sigma_{\underline{C} w} d w$ corresponds to the error $w$, and $\sigma_{\underline{\underline{C}} w} \sigma_{\underline{\underline{C}} w}^{\prime}$ corresponds to $E\left(w^{2}\right)$. The proposition looks a little different because now we choose a vector $v$ rather than a number. However, we could define a residual $\sigma_{\underline{C} w} d w$ and then the problem would reduce to choosing a number, the loading of $d \Lambda$ on this residual. It is not convenient to do so in this case since $\sigma_{\underline{\underline{C}} w}$ potentially changes over time and as state variables change. 
The first term on the right hand side of expression (34) is the market price of risk of the approximate hedge portfolio; since $d z$ and $d w$ are orthogonal, $\sigma_{\underline{C} z}$ is the projection of the $d \underline{C} / \underline{C}$ shocks on the basis asset shocks $d z$. (See equation 25 for market prices of risk.) The $\underline{v}$ term then gives the assignment of $d w$ risk-prices that maximizes or minimizes the asset price drift, subject to the constraints. In the limit that $d \underline{C} / \underline{C}$ shocks are entirely spanned by the basis asset shocks, $\sigma_{\underline{C} w}=0$ and then $\underline{v}=0$.

The only difference between the upper and the lower bound is the sign of $\underline{v}$. At maturity the lower and the upper bound coincide. Before maturity, the lower bound grows with higher drift than the upper bound. We can, in fact write our problem as the maximization (minimization) of the drift of the lower (upper) bound process.

Another way to interpret the maximization is that we assign market prices of risk to the $d w$ shocks to minimize (maximize) the asset price at any date such that the total (sum of squared) market price of risk is bounded. Using equation (30), $v$ is exactly the market price of $d w$ :

$$
-E\left(\frac{d \Lambda}{\Lambda} d w\right)=-E\left[\left(\frac{d \Lambda^{*}}{\Lambda^{*}}-v d w\right) d w\right]=v d t
$$

Equation (31) is just a quadratic constraint on $v$.

As in the discrete-time case, it is worth plugging in the definition of $\Lambda^{*}$ to obtain explicit, if less intuitive, expressions,

$$
\begin{gathered}
\frac{d \underline{\Lambda}}{\underline{\Lambda}}=\tilde{\mu}_{S}^{\prime} \Sigma_{S}^{-1} \sigma_{S} d z-\sqrt{A^{2}-\tilde{\mu}_{S}^{\prime} \Sigma_{S}^{-1} \tilde{\mu}_{S}} \frac{\sigma_{\underline{C} w}}{\sqrt{\sigma_{\underline{C} w} \sigma_{\underline{C} w}^{\prime}}} d w \\
\mu_{\underline{C}}+\frac{x^{c}}{\underline{C}}-r=\tilde{\mu}_{S}^{\prime} \Sigma_{S}^{-1} \sigma_{S} \sigma_{\underline{C} z}+\sqrt{A^{2}-\tilde{\mu}_{S}^{\prime} \Sigma_{S}^{-1} \tilde{\mu}_{S}} \sqrt{\sigma_{\underline{C} w} \sigma_{\underline{C} w}^{\prime}} .
\end{gathered}
$$

The bound at time $t$ is the minimum price of a payoff equal to the bound at $t+\Delta t$; the discount factor to pick depends on what the bound will do at time $t+\Delta t$. In differential form, $\sigma_{\underline{C} w}, \sigma_{\underline{C} z}$ give the behavior of bound at $t+\Delta t$. That is why $\sigma_{\underline{C} w}, \sigma_{\underline{C} z}$ show up in the formula for the discount factor, and why we only derive a restriction between $\sigma_{\underline{C} w}, \sigma_{\underline{C} z}$ and $\mu_{\underline{C}}$ rather than explicit formulas. As in the multiperiod discrete time, case, one has to work backwards from expiration to find the actual value of $C$. 


\subsubsection{Solution 1: a partial differential equation for the price bounds}

We follow two paths to find a solution. First, we find a partial differential equation for the bounds. We hypothesize a solution $\underline{C}(S, V, t)$, we use Ito's lemma to derive expressions for $\mu_{\underline{C}}$ and $\sigma_{\underline{C}}$ and then we substitute in restriction (38) between $\mu_{\underline{C}}$ and $\sigma_{\underline{C}}$. The result is ugly, but straightforward to evaluate numerically.

Proposition 8 The price bound $\underline{C}(S, V, t)$ is the solution to the partial differential equation

$$
\begin{gathered}
\underline{C}_{t}+\left(C_{S_{i}} S\right)^{\prime} \mu_{S}+\underline{C}_{V}^{\prime} \mu_{V}+x^{c}-r C+ \\
+\frac{1}{2} \sum \underline{C}_{S_{i} S_{j}} S_{i} S_{j} \sigma_{S_{i}} \sigma_{S_{j}}^{\prime}+\frac{1}{2} \sum \underline{C}_{V_{i} V_{j}}\left(\sigma_{V z_{i}} \sigma_{V z_{j}}^{\prime}+\sigma_{V w_{j}} \sigma_{V w_{j}}^{\prime}\right)+\sum \underline{C}_{S_{i} V_{j}} S_{i} \sigma_{S_{i}} \sigma_{V z_{j}}^{\prime}= \\
=\tilde{\mu}_{S}^{\prime} \Sigma_{S}^{-1} \sigma_{S} \sigma_{V z}^{\prime} C_{V}+\tilde{\mu}_{S}^{\prime}\left(S C_{S}\right)+\sqrt{A^{2}-\tilde{\mu}_{S}^{\prime} \Sigma_{S}^{-1} \tilde{\mu}_{S}} \sqrt{\underline{C}_{V}^{\prime} \sigma_{V w} \sigma_{V w}^{\prime} \underline{C}_{V}}
\end{gathered}
$$

subject to the boundary conditions provided by the asset payoff $x_{T}^{c}$. Replacing + with - before the square root gives the partial differential equation satisfied by the upper bound.

Proof: We guess that the bound is a twice-differentiable function of state,

$$
\underline{C}=\underline{C}(S, V, t) \text {. }
$$

We have, by Ito's lemma,

$$
\begin{aligned}
d \underline{C}= & \underline{C}_{t} d t+\underline{C}_{S}^{\prime} d S+\underline{C}_{V}^{\prime} d V \\
& +\frac{1}{2} \sum_{i, j} \underline{C}_{S_{i} S_{j}} d S_{i} d S_{j}+\frac{1}{2} \sum_{i, j} \underline{C}_{V_{i} V_{j}} d V_{i} d V_{j}+\sum_{i, j} \underline{C}_{S_{i} V_{j}} d S_{i} d V_{j}
\end{aligned}
$$

where $C_{x}$ denotes $\partial C / \partial x$. Substituting for the $S$ and $V$ processes from equations (26) and (27),

$$
\begin{aligned}
d \underline{C}= & \underline{C}_{t} d t+\left(S \underline{C}_{S}\right)^{\prime}\left(\mu_{S} d t+\sigma_{S} d z\right)+\underline{C}_{V}^{\prime}\left(\mu_{V} d t+\sigma_{V z} d z+\sigma_{V w} d w\right)+ \\
& +\left[\frac{1}{2} \sum_{i, j=1}^{n_{S}} \underline{C}_{S_{i} S_{j}} S_{i} S_{j} \sigma_{S_{i}} \sigma_{S_{j}}^{\prime}+\frac{1}{2} \sum_{i, j=1}^{n_{V}} \underline{C}_{V_{i} V_{j}}\left(\sigma_{V z_{i}} \sigma_{V z_{j}}^{\prime}+\sigma_{V w_{j}} \sigma_{V w_{j}}^{\prime}\right)\right. \\
& \left.+\sum_{i=1}^{n_{S}} \sum_{j=1}^{n_{V}} \underline{C}_{S_{i} V_{j}} S_{i} \sigma_{S_{i}} \sigma_{V z_{j}}^{\prime}\right] d t
\end{aligned}
$$


where $\left(S C_{S}\right)$ indicates element by element multiplication, and $\sigma_{V z_{j}}$ denotes the jth row of $\sigma_{V z}$, etc. Therefore, we can write $\mu_{\underline{C}}$ and $\sigma_{\underline{C} z}, \sigma_{\underline{C} w}$ in terms of derivatives of the $\underline{C}(\cdot)$ function,

$$
\begin{aligned}
& \underline{C} \mu_{\underline{C}}=\underline{C}_{t}+\left(S \underline{C}_{S}\right)^{\prime} \mu_{S}+\underline{C}_{V}^{\prime} \mu_{V}+\frac{1}{2} \sum \underline{C}_{S_{i} S_{j}} S_{i} S_{j} \sigma_{S_{i}} \sigma_{S_{j}}^{\prime} \\
& +\frac{1}{2} \sum \underline{C}_{V_{i} V_{j}}\left(\sigma_{V z_{i}} \sigma_{V z_{j}}^{\prime}+\sigma_{V w_{j}} \sigma_{V w_{j}}^{\prime}\right)+\sum \underline{C}_{S_{i} V_{j}} S_{i} \sigma_{S_{i}} \sigma_{V z_{j}}^{\prime}
\end{aligned}
$$

and

$$
\begin{gathered}
\underline{C} \sigma_{\underline{C} z}=\left(S C_{S}\right)^{\prime} \sigma_{S}+C_{V}^{\prime} \sigma_{V z} \\
\underline{C} \sigma_{\underline{C} w}=C_{V}^{\prime} \sigma_{V w}
\end{gathered}
$$

Hence, we recover the terms needed in equation (38)

$$
\underline{C}^{2} \sigma_{\underline{C} w} \sigma_{\underline{\omega} w}^{\prime}=\underline{C}_{V}^{\prime} \sigma_{V w} \sigma_{V w}^{\prime} \underline{C}_{V}
$$

and

$$
\begin{aligned}
\underline{C} \sigma_{\underline{C} z} \sigma_{S}^{\prime} \Sigma_{S}^{-1} \tilde{\mu}_{S} & =\left[\left(S \underline{C}_{S}\right)^{\prime} \sigma_{S}+\underline{C}_{V}^{\prime} \sigma_{V z}\right] \sigma_{S}^{\prime} \Sigma_{S}^{-1} \tilde{\mu}_{S} \\
& =\left(S \underline{C}_{S}\right)^{\prime} \tilde{\mu}_{S}+\underline{C}_{V}^{\prime} \sigma_{V z} \sigma_{S}^{\prime} \Sigma_{S}^{-1} \tilde{\mu}_{S}
\end{aligned}
$$

Plugging these expressions in restriction (38), we obtain the partial differential equation.

The drift of the price processes of our basis assets $\mu_{S}$ does enter into the partial differential equations that characterize our price bounds. Again, actual and not just risk-neutral probabilities matter.

\subsubsection{Solution 2: a special case in which we know the discount factor.}

In general, the $\Lambda$ process depends on the parameters $\sigma_{\underline{C} w}$. Hence, we cannot use a Monte Carlo integration approach to find the bound: we cannot characterize $\Lambda$ enough to simply calculate

$$
\underline{C}_{t}=E_{t} \int_{s=t}^{T} \frac{\underline{\Lambda}_{s}}{\underline{\Lambda}_{t}} x_{s}^{c} d s+E_{t}\left(\frac{\underline{\Lambda}_{T}}{\underline{\Lambda}_{t}} x_{T}^{c}\right) .
$$

The Feynman-Kac transformation from a partial differential equation into a discount factor does not work here because the partial differential equation is nonlinear. 
However, there is an important special case in which we can find the $\underline{\Lambda}$ process directly. In general the problem is that we do not know how to spread the loading of $d \underline{\Lambda}$ across the multiple sources of risk $d w$ whose risk prices we do not observe. However, if there is only one shock $d w$, then the loading of $d \underline{\Lambda}$ on that risk can be determined simply by the volatility constraint.

Proposition 9 In the special case that there is only one noise dw driving the $V$ process orthogonal to the noises $d z$ that drive the basis assets $S$, we can find the optimum discount factor $\underline{\Lambda}$ directly as

$$
\frac{d \underline{\Lambda}}{\underline{\Lambda}}=-r d t-\tilde{\mu}_{S}^{\prime} \Sigma_{S}^{-1} \sigma_{S} d z-\sqrt{A^{2}-\tilde{\mu}_{S}^{\prime} \Sigma_{S}^{-1} \tilde{\mu}_{S}} d w
$$

Proof. In the special case, $d w$ and $\sigma_{\underline{C} w}$ are scalars. Hence equation (33) simplifies as given.

This expression does completely characterize $d \underline{\Lambda}$. This approach is useful in situations with many state variables. For example, Aït-Sahalia, Cochrane and Saá-Requejo (1995) price term structure derivatives. There is only one non-priced shock, the short rate, but the yields on bonds of every maturity are state variables. Solving a high-dimensional partial differential equation numerically is impractical, but one can find price bounds for any given value of the state variables by Monte Carlo integration.

\subsection{Pricing a call option with stochastic stock volatility and in- terest rates}

Stochastic volatility models can produce a rich variety of pricing effects compared with BlackScholes model and are used to explain many observed anomalies relative to that model. For example, stochastic volatility of the underlying asset increases the kurtosis of underlying returns, which can generate the "volatility smile," or apparently high prices of out of the money options. If we allow correlation between volatility and the underlying price - for example if price declines set off a period of high volatility - then we induce skewness in the underlying asset return distribution, which can generate a volatility "smirk" — the fact that out-of-the-money options are more "overpriced" than in-the-money options. Some examples 
of this literature are Hull and White (1987), Stein and Stein (1991), Heston (1994) and Bates (1995). In each case, these models assume a value for the market price of volatility risk, and then pretend to price by arbitrage.

\subsection{A stochastic-volatility stochastic-interest-rate model}

We posit a standard stochastic volatility model ${ }^{17}$, with a stochastic instantaneous interest rate and potentially correlated shocks:

$$
\begin{aligned}
\frac{d S}{S} & =\mu_{S}(\cdot) d t+\sqrt{V} d z_{S} \\
d V & =\alpha_{V}(\bar{V}-V) d t+\beta_{S} \sqrt{V} d z_{S}+\beta_{V} \sqrt{V} d z_{V} \\
d r & =\alpha_{r}(\bar{r}-r) d t++\sigma_{S} \sqrt{r} d z_{S}+\sigma_{V} \sqrt{r} d z_{V}+\sigma_{r} \sqrt{r} d z_{r}
\end{aligned}
$$

where $d z_{S}, d z_{V}$ and $d z_{r}$ are independent Brownian increments. We can impose different correlation patterns between the three processes by choice of the $\beta, \sigma$ coefficients. We recover the special case studied by Hull and White (1987) and Stein and Stein (1991), in which the volatility is uncorrelated with the stock price, by setting $\beta_{S}=0$. A constant interest rate results from with $\sigma_{r}=\sigma_{V}=\sigma_{S}=0$. A constant interest rate assumption, common in the stochastic volatility literature, is convenient because one only has one extra unpriced shock. Conversely, the varying interest rate case is important to show how our technique can handle multiple sources of nontradable uncertainty.

We pursue the partial differential equation approach. We posit that the solution is a function of state variables,

$$
C=C(S, V, r, t)
$$

Specializing proposition 8 to this stochastic volatility model and simplifying slightly, the price function must satisfy the ugly partial differential equation

$$
\begin{aligned}
& \quad C_{t}+C_{S} S r+C_{V}\left[\alpha_{V}(\bar{V}-V)-\beta_{S}\left(\mu_{S}-r\right)\right]+C_{r}\left[\alpha_{r}(\bar{r}-r)-\left(\mu_{S}-r\right) \sigma_{S} \sqrt{\frac{r}{V}}\right]+ \\
& +\frac{1}{2}\left[C_{S S} S^{2} V+C_{V V}\left(\beta_{S}^{2}+\beta_{V}^{2}\right) V+C_{r r}\left(\sigma_{S}^{2}+\sigma_{V}^{2}+\sigma_{r}^{2}\right) r\right] \\
& +C_{S V} \beta_{S} S V+C_{S r} \sigma_{S} S \sqrt{r V}+C_{V r}\left(\beta_{S} \sigma_{S}+\beta_{V} \sigma_{V}\right) \sqrt{r V}-r C \\
& = \pm \sqrt{A^{2}-\frac{\left(\mu_{S}-r\right)^{2}}{V}} \sqrt{\left(C_{V} \beta_{V} \sqrt{V}+C_{r} \sigma_{V} \sqrt{r}\right)^{2}+C_{r}^{2} \sigma_{r}^{2} r}
\end{aligned}
$$

\footnotetext{
${ }^{17}$ Our model for the volatility is similar to Heston (1994) and Stein and Stein (1991).
} 
subject to the usual boundary conditions,

$$
C\left(S_{T}, V, r, T\right)=\max \left(S_{T}-K, 0\right) ; C(0, V, r, t)=0
$$

We solve this equation numerically. The explicit method for numerically solving a partial differential equation of this sort starts with values of $C(S, V, r, t)$ on a grid for values of $S, V, r$ at time $t$ - for example at the boundary condition of expiration. One takes numerical partial derivatives $C(S, V, r, t)$ with respect to $S, V, r$. Then equation (39) can be solved for $C_{t}$ at each value of the $S, V, r$ grid. Finally, we obtain a new value $C(S, V, r, t-\Delta t)=C(S, V, r, t)-C_{t} \Delta t$.

The implicit method for solving a partial differential equation seeks to simultaneously search for the new value $C(S, V, r, t-\Delta t)$ and the partial derivatives of $C(S, V, r, t-\Delta t)$ with respect to $S, V, r$ at time $t-\Delta t$ that generate the value of $C(S, V, r, t)$. Implicit numerical methods are often more stable - small errors do not propagate so easily - and thus can converge faster and on a coarser grid than explicit methods. (See Brennan and Schwartz 1978 for a discussion of numerical methods for solving partial differential equations in financial applications.)

However, the non-linear nature of the partial differential equation makes it difficult to apply a fully implicit method The nonlinearity means that the simultaneous solution of the value of $C(S, V, r, t-\Delta t)$ and its partial derivatives (nearby values) that generate $C(S, V, r, t)$ is a set of nonlinear equations, where usually this step just requires a matrix inversion.

Therefore, we pursue instead a fully explicit method, as described above. This method is easier to program, and it does converge, albeit more slowly than an implicit or mixed implicit-explicit method might. We use a very fine time grid $\Delta t=0.00005$ year (very fine!), a stock grid from $\$ 10$ to $\$ 500$ spaced $\$ 5$, a volatility (standard deviation units) grid from 0.1 to 0.3 spaced 0.01 , and an interest rate grid from 0.01 to 0.11 spaced 0.02 . We smooth second derivatives by averaging the values for three adjacent grid points.

\subsection{Results}

Table 3 presents the parameter values for our stochastic volatility process. We do not attempt a formal estimation, but just try to pick reasonable values for an illustrative calculation. Our values are roughly consistent with the stochastic volatility literature, such as Stein and Stein 
(1991), Heston (1994). We pick a drift process for the stock

$$
\mu_{S}=0.5 \sqrt{V}+r
$$

This choice gives a constant instantaneous Sharpe ratio for the basis assets of $\left(\mu_{S}-r\right) / \sqrt{V}=$ 0.5 . We start with $\beta_{S}=\sigma_{V}=\sigma_{S}=0$, or no correlation between the shocks. $\beta_{V}$ then drives the volatility of volatility, we use Stein and Stein's (1991) estimate. $\bar{r}$ and $\bar{V}$ drive the mean short rate and volatility. We pick these parameters to produce a $5 \%$ mean interest rate and $16 \%$ mean stock volatility as in Table 2 . The $\alpha^{\prime} s$ describe the speed of mean-reversion in volatility and short rate. Our values give a little less than a year half-life to volatility and the short rate.

Table 3: Parameters used for stochastic volatility-stochastic interest rate model.

\begin{tabular}{r|cccccc} 
Parameter: & $\beta_{V}$ & $\sigma_{r}$ & $\alpha_{V}$ & $\sqrt{\bar{V}}$ & $\alpha_{r}$ & $\bar{r}$ \\
\hline Value: & 0.5 & 0.18 & 1.4 & 0.1409 & 1.214 & 0.0488
\end{tabular}

Parameters for stochastic volatility/stochastic interest rate call option calcluations. The model is

$$
\begin{gathered}
\frac{d S}{S}=\mu_{S}(\cdot) d t+\sqrt{V} d z_{S} \\
d V=\alpha_{V}(\bar{V}-V) d t+\beta_{V} \sqrt{V} d z_{V} \\
d r=\alpha_{r}(\bar{r}-r) d t+\sigma_{r} \sqrt{r} d z_{r} \\
\mu_{S}(\cdot)=0.5 \sqrt{V}+r .
\end{gathered}
$$

Figures 8 and 9 present some price bound calculations, and contrast the price bounds with the Black-Scholes model and standard stochastic-volatility models. As above, we calculate price bounds with a target Sharpe ratio twice that of the market, or $h=1.0$. Option prices depend on the two state variables in addition to the stock price, volatility $V$ and the short rate $r$. The figures present option prices at the means of the state variables, $V=\bar{V}, r=\bar{r}$. To aid comparison we plot the difference between the option price bound and the BlackScholes price, using a volatility parameter $\bar{V}$ in the Black-Scholes formula. The figures also include the option prices (difference relative to Black-Scholes) calculated using the standard assumption of a zero market price of volatility risk.

Figure 8 presents price bounds for one year call options with a constant interest rate equal to $\bar{r}$. If we assume a zero market price of volatility risk, we reproduce the standard 
volatility smile prediction in the near-money region. However, the option price bounds are fairly loose around the Black Scholes price, especially for near-money options. The volatility smile all lies within our bounds! This finding suggests that the volatility smile is not a robust feature of the stochastic volatility model, but depends crucially on the assumed value of the market price of volatility risk.

Figure 9 adds a stochastic interest rate as well as stochastic volatility. Option price bounds are again loose around the Black Scholes price, and the volatility smile is not "significant." However, now the bounds are large for far-in-the money options at the right end of the graph as well as for nearly at the money options as in Figure 8. At-the money options are very sensitive to changes in volatility, because in this area the curvature of the payoff as a function of underlying asset price is highest. Far in the money options are much more sensitive to interest rates, since their payoff is essentially replicated by holding the underlying asset. Therefore, our bounds assign most of the total price of risk to interest rate risk if we are analyzing far-in-the money options and to volatility risk if we are analyzing around-the money options. 


\section{Conclusions and Extensions}

\section{Statistical model}

In the numerical examples presented here, we use highly parametric models of the conditional distributions of our basis assets. These assumptions contrast our results to the existing option pricing literature, which exploits simple models in order to use arbitrage arguments or obtain closed form solutions.

But we have abandoned arbitrage, and our bounds resist closed-form solutions. Hence, there is little reason to insist on tight parametric models for the underlying and basis assets. "Non-parametric," "Semi-parametric" or discrete models of the conditional distribution or drift and diffusion coefficients of our basis assets and state variables are more natural models for practice, as we used in evaluating multiple trading dates.

The Sharpe ratios generated by the basis assets are a crucial element of our bounds. Statistical models for option pricing typically focus on estimating volatilities, and can do a bad job of estimating means. They can lead to implausible estimates of conditional Sharpe ratios. One must be careful to specify models for the distribution of underlying assets that produce reasonable Sharpe ratios.

Tightening the bounds: more assets.

Since the size of our bounds is directly related to the $R^{2}$ in a regression of option payoffs on basis asset payoffs, we are led away from the usual option pricing practice of specifying only a few hedge assets, and toward using as wide a variety of hedge assets as possible, to increase the $R^{2}$ and hence tighten the bounds. The difficulty, of course, is building a reasonable model of the joint distribution of large numbers of assets, and (as always) not spuriously raising $R^{2}$.

The size of our bounds is also directly related to the difference between the target Sharpe ratio and the Sharpe ratio on the basis assets. This fact suggests that one include basis assets with high Sharpe ratios to obtain tighter bounds. For example, one might include a market portfolio along with the underlying stock to hedge an individual stock option.

Tightening the bounds: more discount factor restrictions. 
A natural way to get tighter bounds is to add restrictions on the space of discount factors. We can think of three types of constraint that may be immediately useful and practical.

First, the "stochastic dominance" literature ( Levy 1985, Constantinides 1994) calculates option price bounds under the assumption that the discount factor is monotonic in a state variable. We have a related somewhat weaker condition already: the covariance between basis asset returns and discount factor must be negative to generate positive expected returns (the pricing constraint). But global monotonicity is more stringent than covariance, and this constraint may tighten the bounds.

Second, the price bounds make the worst-case assumption that the discount factor is perfectly correlated with a portfolio of basis assets plus the option in question. A limit on the correlation between discount factors and asset payoffs will thus sharpen the bounds for a given discount factor variance target. This limit makes sense when the basis assets are known to be inefficient (bonds for pricing swaps and caps, individual securities when pricing their options, rather than index options).

Third, it is useful to consider many assets at once in calibrating our model, rather than just the few assets common in replication arguments. For example, using bond data to limit assumptions about the market price of interest rate risk in a stock option model can sharpen pricing bounds. Rather than add a model for the joint time-series evolution of bonds, one might include such information more informally, by further reducing the set of allowable discount factors. For example, we could add to our stochastic volatility - stochastic interest rate calculation the stipulation that the discount factor generate less than a given market price of interest rate risk, by the condition $\left|E\left(d \Lambda / \Lambda \sigma_{r} d z_{r}\right)\right| \leq B$. 


\section{Appendix}

\subsection{Evaluating expectations with positive discount factors and lognormal returns.}

We have to evaluate the integral

$$
E\left\{\left[-\frac{x^{c}+\lambda^{\prime} \mathbf{x}}{\delta}\right]^{+2}\right\}=\int_{0}^{\infty} \max \left[-\frac{S \max \left(R-\frac{K}{S}, 0\right)+\lambda_{0} R^{f}+\lambda_{1} R}{\delta}, 0\right]^{2} f(R) d R
$$

Taking only the part of the integral where the outer max operator is positive, and breaking up the integral according to the value of the interior max, we obtain

$$
\begin{gathered}
=\frac{1}{\delta^{2} \int} \int_{\frac{\left(\lambda_{0} R^{f}-K\right)+\left(\lambda_{1}+S\right) R}{\delta}<0, R>\frac{K}{S}}\left[\left(\lambda_{0} R^{f}-K\right)+\left(\lambda_{1}+S\right) R\right]^{2} f(R) d R+ \\
\frac{1}{\delta^{2}} \int_{\frac{\lambda_{0} R^{f}+\lambda_{1} R}{\delta}<0, R<\frac{K}{S}}\left[\lambda_{0} R^{f}+\lambda_{1} R\right]^{2} f(R) d R .
\end{gathered}
$$

Now we express the limits on the integrals as ranges for the random variable $R$. The result depends on the signs of the terms multiplying $R$ in the integrals. For the first integral we have

$$
\left\{\begin{array}{cc}
\frac{\lambda_{1}+S}{\delta}>0: & \frac{K}{S}<R<\frac{K-\lambda_{0} R^{f}}{\lambda_{1}+S} \\
\frac{\lambda_{1}+S}{\delta}<0 & \max \left(\frac{K}{S}, \frac{K-\lambda_{0} R^{f}}{\lambda_{1}+S}\right)<R<\infty
\end{array}\right.
$$

Similarly, the range for the second interval is

$$
\left\{\begin{array}{cc}
\frac{\lambda_{1}}{\delta}>0: & 0<R<\min \left(\frac{K}{S},-\frac{\lambda_{0} R^{f}}{\lambda_{1}}\right) \\
\frac{\lambda_{1}}{\delta}<0: & -\frac{\lambda_{0} R^{f}}{\lambda_{1}}<R<\frac{K}{S}
\end{array}\right.
$$

Given these ranges, we use the fact that for $R \sim \mathcal{N}\left(\mu T, \sigma^{2} T\right)$, we have

$$
\begin{gathered}
\int_{a}^{b}(c+d R)^{2} f(R) d R= \\
=c^{2}\left[\Phi\left(b^{*}\right)-\Phi\left(a^{*}\right)\right]+2 c d e^{\left(\mu+\frac{\sigma^{2}}{2}\right)}\left[\Phi\left(b^{*}-\sigma \sqrt{T}\right)-\Phi\left(a^{*}-\sigma \sqrt{T}\right)\right]+ \\
+d^{2} e^{2\left(\mu+\sigma^{2}\right)}\left[\Phi\left(b^{*}-2 \sigma \sqrt{T}\right)-\Phi\left(a^{*}-2 \sigma \sqrt{T}\right)\right]
\end{gathered}
$$

where

$$
a^{*}=\frac{\ln a-\mu T}{\sigma \sqrt{T}}, b^{*}=\frac{\ln b-\mu T}{\sigma \sqrt{T}} .
$$


Now we can evaluate the expectation in equation (16) for any value of $\lambda, \delta$, so we can search for $\lambda, \delta$ to minimize or maximize the option value.

\subsection{Multiple option integrals}

For the case where we are allowed to trade in $N-1$ options ( $N$ options when we include the option we are trying to price), we write

$$
\begin{gathered}
E\left\{\left[-\frac{x^{c}+\lambda^{\prime} \mathbf{x}}{\delta}\right]^{+2}\right\}= \\
=\int_{0}^{\infty} \max \left[-\frac{\sum_{i=1}^{N} \lambda_{i} S \max \left(R-\frac{K_{i}}{S}, 0\right)+\lambda_{N+1} R^{f}+\lambda_{N+2} R}{\delta}, 0\right]^{2} f(R) d R
\end{gathered}
$$

where $\lambda_{i}=1$ if the index $i$ refers to the option we are trying to price and where without loss of generality we assume that

$$
K_{1}<K_{2}<\cdots<K_{N}
$$

Taking only the part of the integral where the max operator is positive,

$$
=\int_{D<0}^{\infty} \max \left[\frac{\sum_{i=1}^{N} \lambda_{i} S \max \left(R-\frac{K_{i}}{S}, 0\right)+\lambda_{N+1} R^{f}+\lambda_{N+2} R}{\delta}\right]^{2} f(R) d R
$$

where

$$
D=\frac{\sum_{i=1}^{N} \lambda_{i} S \max \left(R-\frac{K_{i}}{S}, 0\right)+\lambda_{N+1} R^{f}+\lambda_{N+2} R}{\delta}
$$

Now we break up the integral successively according to the value of the interior maximizations, starting by that corresponding to the option with the largest strike

$$
\begin{gathered}
=\frac{1}{\delta^{2}} \int_{D<0}^{\infty} \max \left[\frac{\sum_{i=1}^{N} \lambda_{i} S \max \left(R-\frac{K_{i}}{S}, 0\right)+\lambda_{N+1} R^{f}+\lambda_{N+2} R}{\delta}\right]^{2} f(R) d R \\
=\frac{1}{\delta^{2}} \int_{L_{N}<0, R>\frac{K_{N}}{S}}\left[\left(\lambda_{N+1} R^{f}-\sum_{j=1}^{N} \lambda_{j} K_{j}\right)+\left(\lambda_{N+2}+\sum_{j=1}^{N} \lambda_{j} S\right) R\right]^{2} f(R) d R+ \\
+\cdots+\frac{1}{\delta^{2}} \int_{L_{i}<0, R>\frac{K_{i}}{S}, R<\frac{K_{i+1}}{S}}\left[\left(\lambda_{N+1} R^{f}-\sum_{j=1}^{i} \lambda_{j} K_{j}\right)+\left(\lambda_{N+2}+\sum_{j=1}^{i} \lambda_{j} S\right) R\right]^{2} f(R) d R+ \\
+\cdots+\frac{1}{\delta^{2}} \int_{\frac{\lambda_{N+1} R^{f}+\lambda_{N+2} R}{\delta}<0, R<\frac{K_{1}}{S}}\left[\lambda_{N+1} R^{f}+\lambda_{N+2} R\right]^{2} f(R) d R
\end{gathered}
$$


where

$$
\begin{aligned}
L_{n} & =\frac{\left(\lambda_{N+1} R^{f}-\sum_{j=1}^{N} \lambda_{j} K_{j}\right)+\left(\lambda_{N+2}+\sum_{j=1}^{N} \lambda_{j} S\right) R}{\delta} \\
L_{i} & =\frac{\left(\lambda_{N+1} R^{f}-\sum_{j=1}^{i} \lambda_{j} K_{j}\right)+\left(\lambda_{N+2}+\sum_{j=1}^{i} \lambda_{j} S\right) R}{\delta}
\end{aligned}
$$

We express the limits on the integrals as ranges for the random variable $R$. Since one inverts $<$ and $>$ operators when multiplying by a negative number, the result depends on the signs of the terms multiplying $R$ in the integrals. For the first integral we have

$$
\left\{\begin{array}{cc}
\frac{\lambda_{N+2}+\sum_{j=1}^{N} \lambda_{j} S}{\delta}>0: & \frac{K_{N}}{S}<R<\frac{-\lambda_{N+1} R^{f}+\sum_{j=1}^{N} \lambda_{j} K_{j}}{\lambda_{N+2}+\sum_{j=1}^{N} \lambda_{j} S} \\
\frac{\lambda_{N+2}+\sum_{j=1}^{N} \lambda_{j} S}{\delta}<0: & \max \left(\frac{-\lambda_{N+1} R^{f}+\sum_{j=1}^{N} \lambda_{j} K_{j}}{\lambda_{N+2}+\sum_{j=1}^{N} \lambda_{j} S}, \frac{K_{N}}{S}\right)<R<\infty
\end{array}\right.
$$

Similarly, for the i-th integral we have

$$
\begin{cases}\frac{\lambda_{N+2}+\sum_{j=1}^{i} \lambda_{j} S}{\delta}>0: & \frac{K_{i}}{S}<R<\min \left(\frac{-\lambda_{N+1} R^{f}+\sum_{j=1}^{i} \lambda_{j} K_{j}}{\lambda_{N+2}+\sum_{j=1}^{i} \lambda_{j} S}, \frac{K_{i+1}}{S}\right) \\ \frac{\lambda_{N+2}+\sum_{j=1}^{i} \lambda_{j} S}{\delta}<0: & \max \left(\frac{-\lambda_{N+1} R^{f}+\sum_{j=1}^{i} \lambda_{j} K_{j}}{\lambda_{N+2}+\sum_{j=1}^{i} \lambda_{j} S}, \frac{K_{i}}{S}\right)<R<\frac{K_{i+1}}{S}\end{cases}
$$

and finally for the last integral

$$
\left\{\begin{array}{cc}
\frac{\lambda_{N+2}}{\delta}>0: & 0<R<\min \left(\frac{K_{1}}{S},-\frac{\lambda_{N+1} R^{f}}{\lambda_{N+2}}\right) \\
\frac{\lambda_{N+2}}{\delta}<0: & -\frac{\lambda_{N+1} R^{f}}{\lambda_{N+2}}<R<\frac{K_{1}}{S}
\end{array}\right.
$$

Given these ranges, we can evaluate the expectation in equation (16) for any value of $\lambda, \delta$, so we can search for $\lambda, \delta$ to minimize or maximize the option value. 


\section{References.}

Aït-Sahalia, Yacine, John H. Cochrane and Jesús Saá-Requejo, 1995, "Good-Deal Pricing of a Term Structure Derivative," Manuscript, University of Chicago

Bates David, 1995, "Post-87 Crash Fears in S\&P 500 Futures Options", The Wharton School working paper.

Bertsekas, David and Steve Shreve, 1978, Stochastic Optimal Control, The Discrete Time Case, New York: Academic Press

Black, Fischer and Myron Scholes, 1973, "The Valuation of Options and Corporate Liabilities," Journal of Political Economy, 81, 637-654.

Brennan, Michael and Eduardo Schwartz, 1978, "Finite Difference Methods and Jump Processes Arising in the Pricing of Contingent Claims: A Synthesis," Journal of Financial and Quantitative Analysis, 13, 462-474.

Chen, Ren-Raw and Louis Scott, 1993, "Maximum Likelihood Estimation for a Multifactor Equilibrium Model of the Term Structure of Interest Rates," Journal of Fixed Income, 14-31.

Cochrane, John H., and Lars Peter Hansen 1992, "Asset Pricing Explorations for Macroeconomics," in Olivier Blanchard and Stanley Fischer eds., 1992 NBER Macroeconomics Annual 115-165.

Constantinides, George M., 1994, "Option Pricing Bounds Based on Stochastic Dominance," Working paper, Graduate School of Business, University of Chicago.

Constantinides, George M. and Thaleia Zariphopoulou, 1995, "Universal Bounds on Option Prices with Proportional Transaction Costs," Working paper, Graduate School of Business, University of Chicago.

Fama, Eugene F. and Kenneth R. French, 1993, "Common Factors in the Returns on Stocks and Bonds," Journal of Financial Economics, 33 3-56. 
Hansen, Lars Peter and Scott Richard, 1987, "The Role of Conditioning Information in Deducing Testable Restrictions Implied by Dynamic Asset Pricing Models," Econometrica, 55, 587-614.

Hansen, Lars Peter and Ravi Jagannathan, 1991, "Implications of Security Market Data for Models of Dynamic Economies". Journal of Political Economy, 99, 225-262.

Hansen, Lars Peter, John Heaton and Erzo Luttmer, 1995, " Econometric Evaluation of Asset Pricing Models" The Review of Financial Studies, 8, 237-274.

Harrison, J. Michael and David Kreps, 1979, "Martingales and Arbitrage in Multiperiod Securities Markets," Journal of Economic Theory, 20, 38I-408.

He, Hua and Neil Pearson, 1992, "Consumption and Portfolio Policies with Incomplete Markets: The Infinite Dimensional Case," Journal of Economic Theory, 54, 259-305.

Heston, Steven ,1994, "A Closed-Form Solution for Options with Stochastic Volatility with Applications to Bond and Currency Options," The Review of Financial Studies, 6, 327-343.

Hull, John C. and Allan White, 1987, "The Pricing of Options on Assets with Stochastic Volatilities," Journal of Finance, 42, 281-300.

Karatzas, Ioannis, John Lehoczky, Steven Shreve and Gan-Lin Xu, 1991, "Martingale and Duality Methods for Utility Maximization in an Incomplete Market," SIAM Journal of Control and Optimization, 29, 702-730.

Levy, Haim, 1985, "Upper and Lower Bounds of Put and Call Option Value: Stochastic Dominance Approach," Journal of Finance, 40, 1197-1217.

MacKinlay, A. Craig, 1995, "Multifactor Models Do Not Explain Deviations from the CAPM," Journal of Financial Economics, 38, 3-28.

Mehra, Rajnish, and Edward C. Prescott, 1985, "The Equity Premium: A Puzzle," Journal of Monetary Economics, 15, 145-161.

Merton, Robert C. 1973a "An intertemporal Capital Asset Pricing Model," Econometrica $41,867-87$. 
Merton, Robert C., 1973b, "Theory of Rational Option Pricing," Bell Journal of Economics and Management Science," 4, 141-83

Ritchken, Peter H., 1985, “On Option Pricing Bounds," Journal of Finance, 40, 1219-1233.

Ross, Steven,1976 "Options and Efficiency," Quarterly Journal of Economics, 90, 75-89.

Ross, Steven, 1976 "The Arbitrage Pricing Theory of Capital Asset Pricing," Journal of Economic Theory, 13, 341-60.

Rubinstein, Mark, 1976, "The Valuation of Uncertain Income Streams and the Pricing of Options," The Bell Journal of Economics, 7, 407-425.

Rubinstein, Mark, 1994, "Presidential Address: Implied Binomial Trees, " Journal of Finance, 49, 771-818.

Stein, Elias.M. and Jeremy C. Stein, 1991, "Stock Price Distributions with Stochastic Volatility: An Analytic Approach," The Review of Financial Studies, 4, 727-752. 


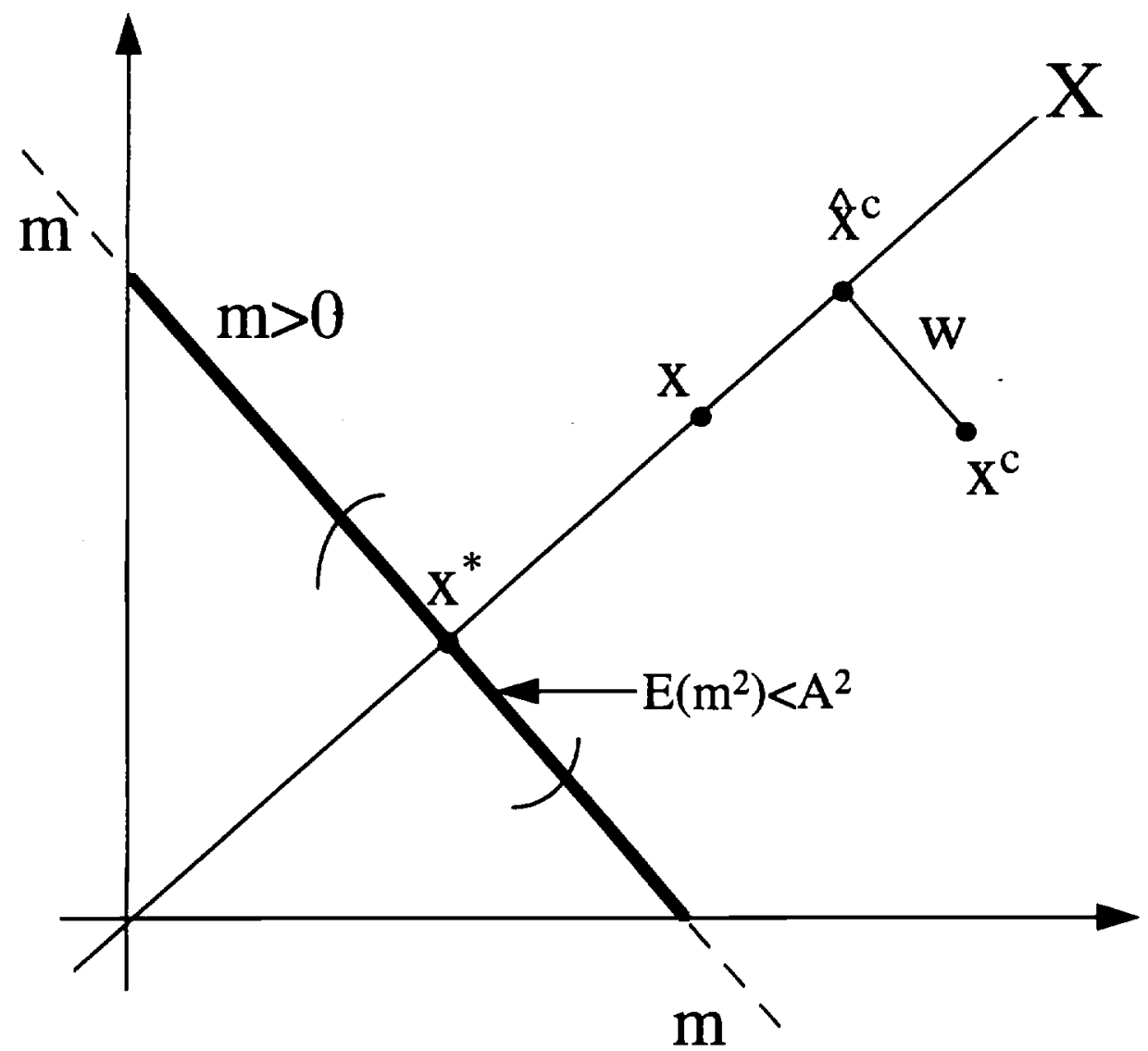

$$
\begin{array}{lll}
X=\text { space of payoffs } & \ldots & \mathrm{m}: \mathrm{p}=\mathrm{E}(\mathrm{mx}), \mathrm{x} \text { in } \mathrm{X} \\
\mathrm{x}=\text { basis payoffs } & \mathrm{m}: \mathrm{m}>0 \\
\mathrm{x}^{\mathrm{c}}=\text { derivative payoff } & \left(\quad \mathrm{m}: \mathrm{E}\left(\mathrm{m}^{2}\right)<\mathrm{A}^{2}\right.
\end{array}
$$

Figure 1: Notation and geometry 
Call price bounds as function of stock price, $K=100$

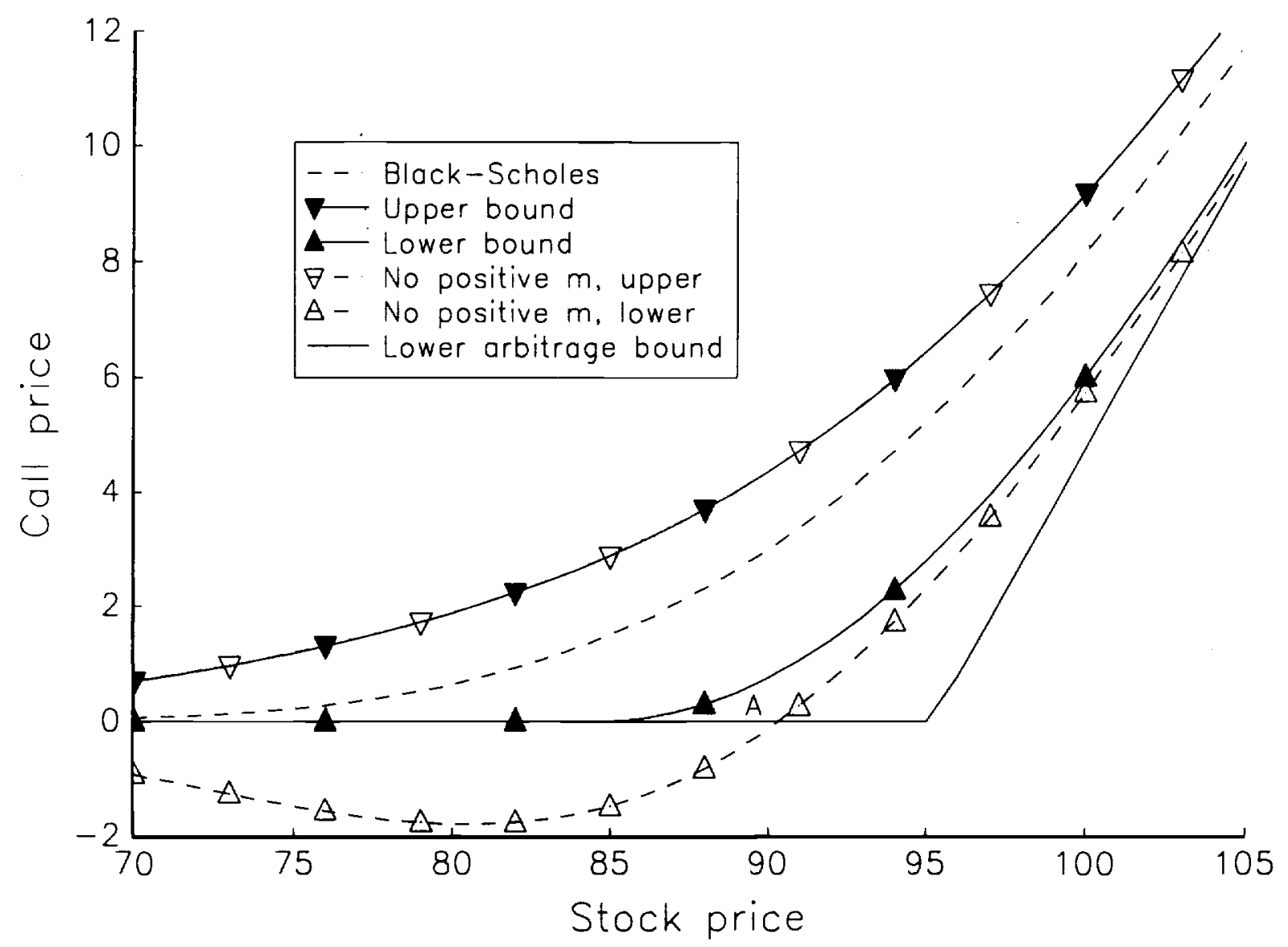

Figure 2: Option price bounds as a function of stock price. Options have one year to expiration and strike price $K=\$ 100$. The bounds assume no trading until expiration, and a target Sharpe ratio equal to twice the market Sharpe ratio, or $h=1.0$. The stock is lognormally distributed with parameters given in table 2 


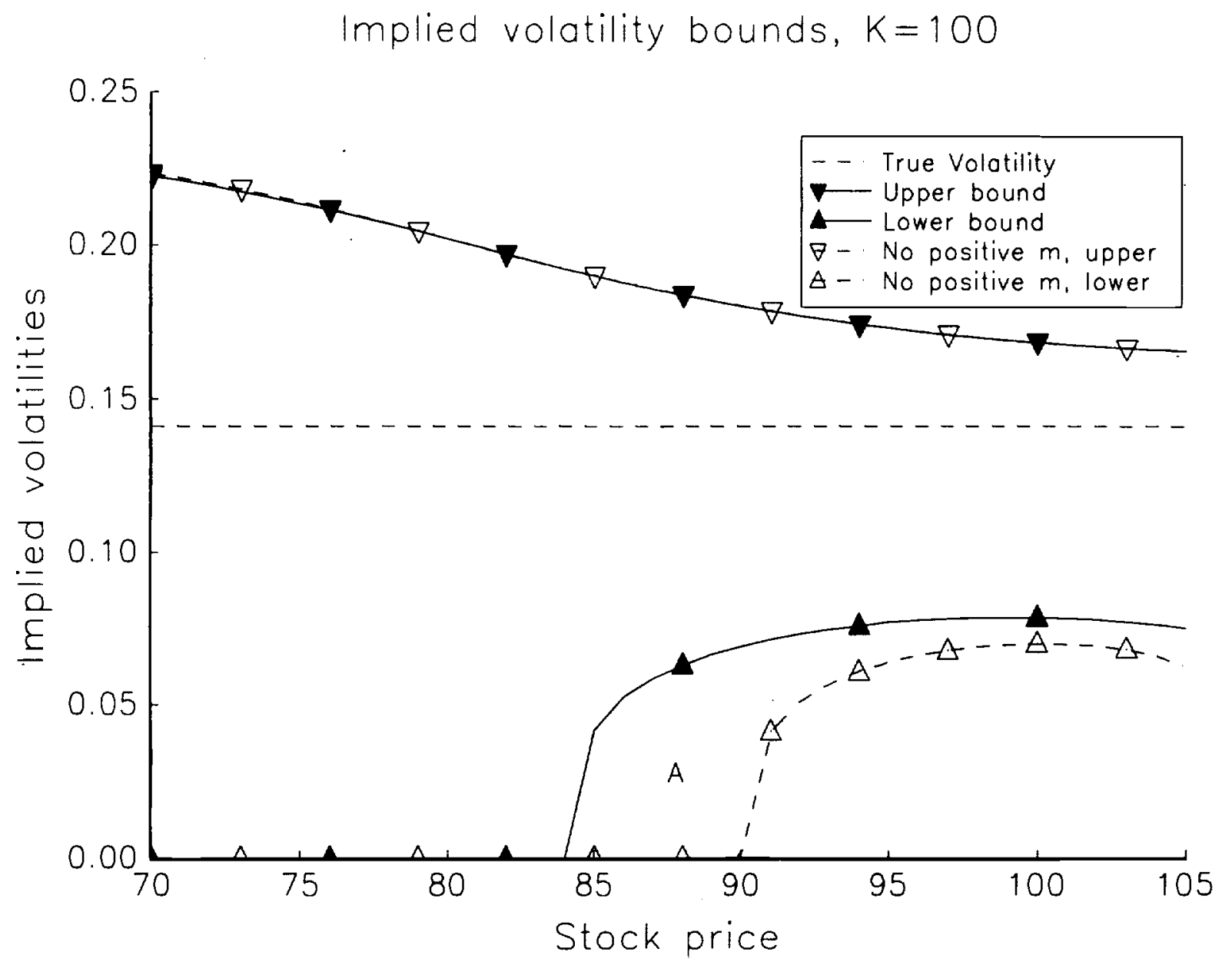

Figure 3: Implied volatilities of upper and lower option pricing bounds. Same calculations as in figure 2 . 


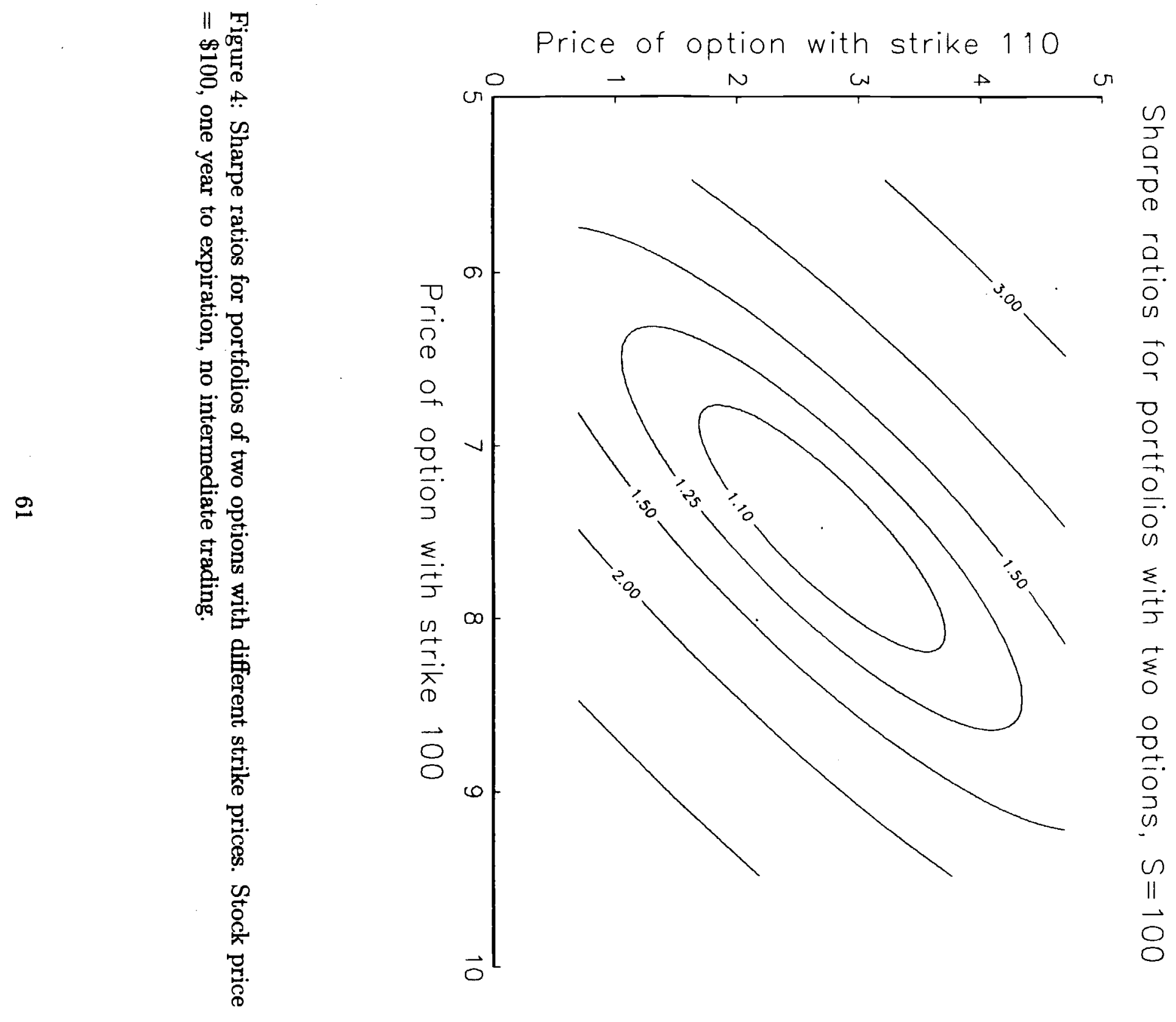


Option price bounds, $S=100$

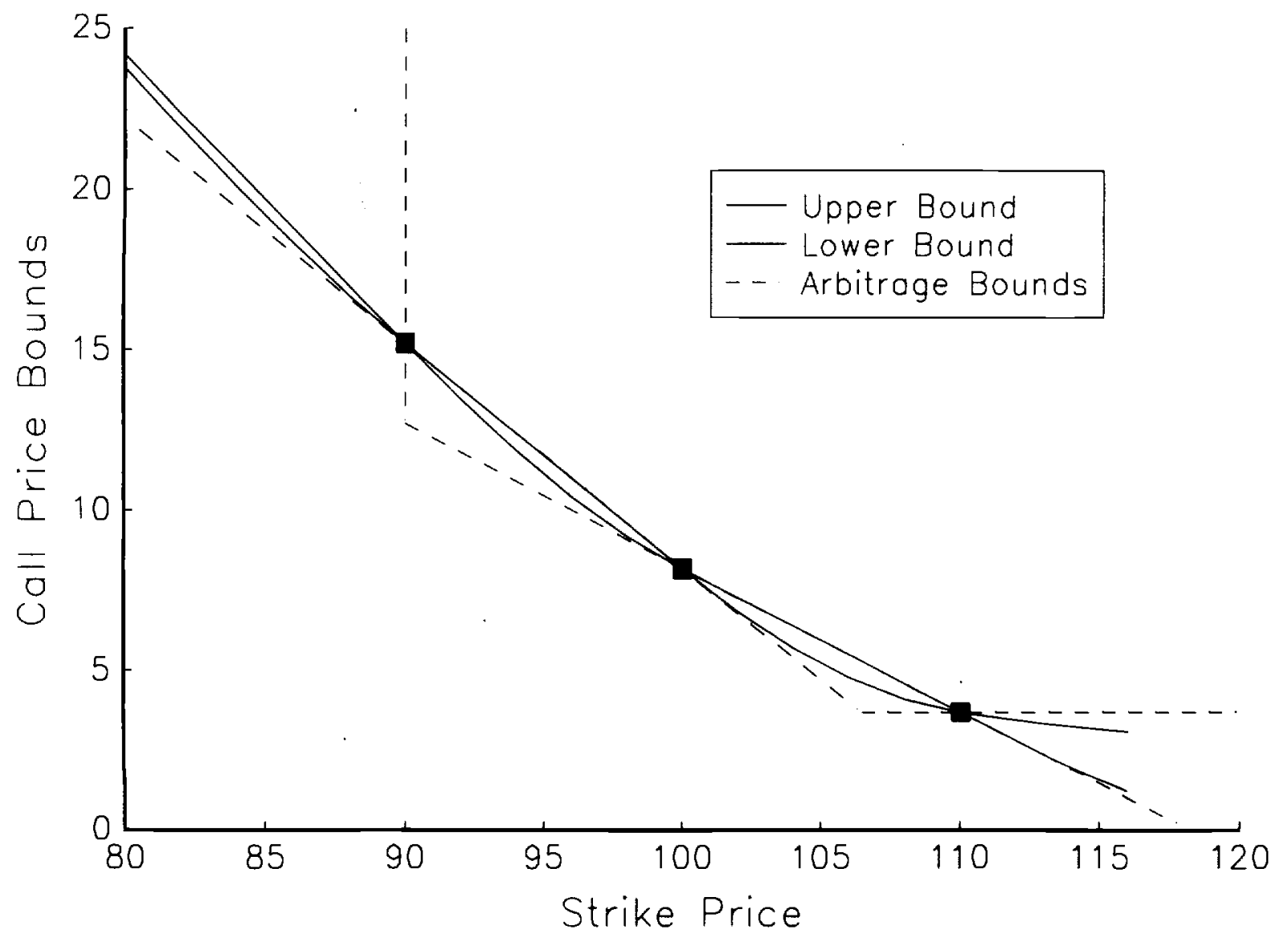

Figure 5: Bounds on option prices with one year to expiration, no intermediate trading. Options are hedged with stock, interest rate, and 3 options whose prices and strikes are marked with squares. 
Option delto bounds, $S=100$

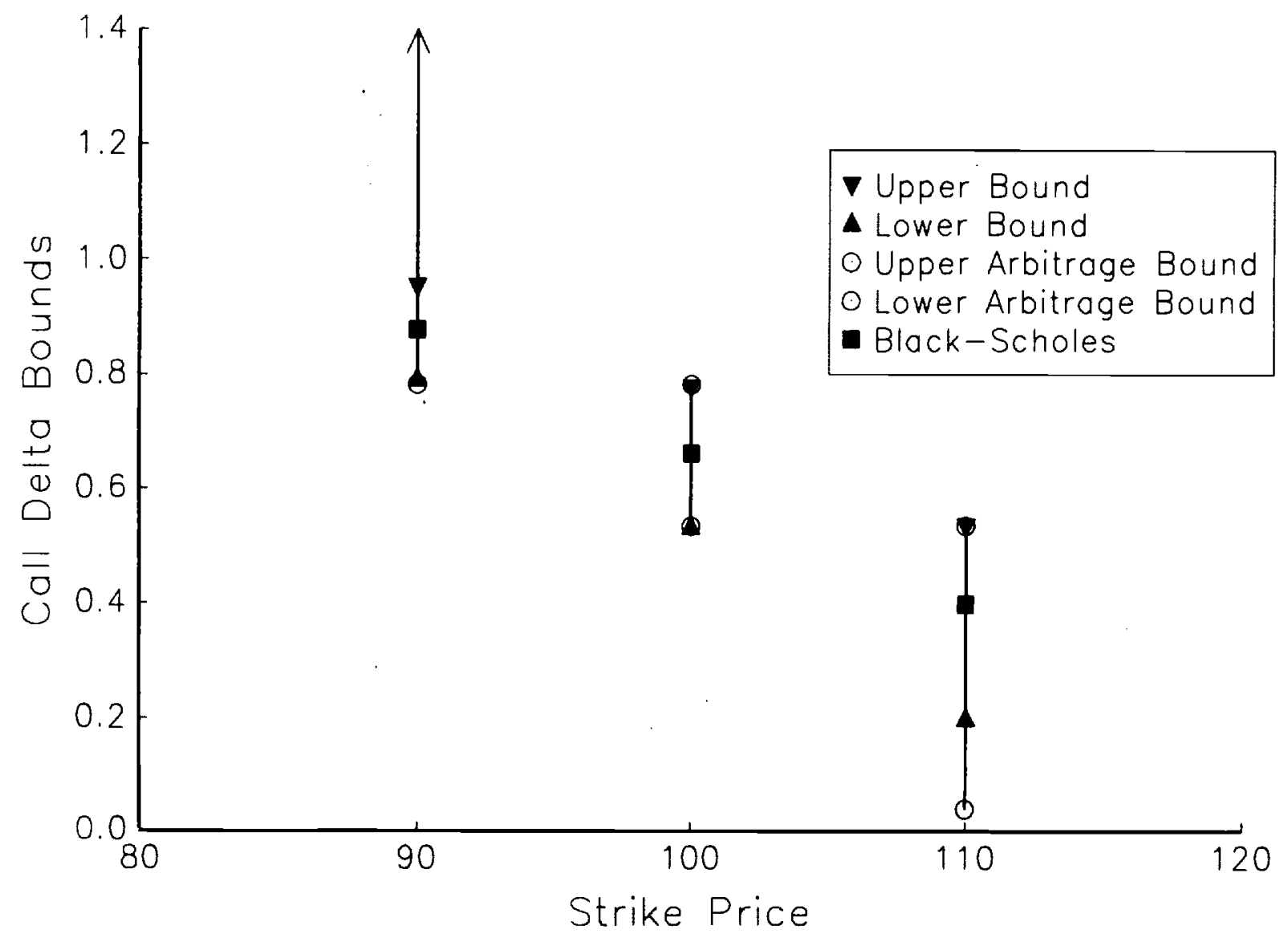

Figure 6: Bounds on option prices deltas $\partial C / \partial S$ with one year to expiration, no intermediate trading. 


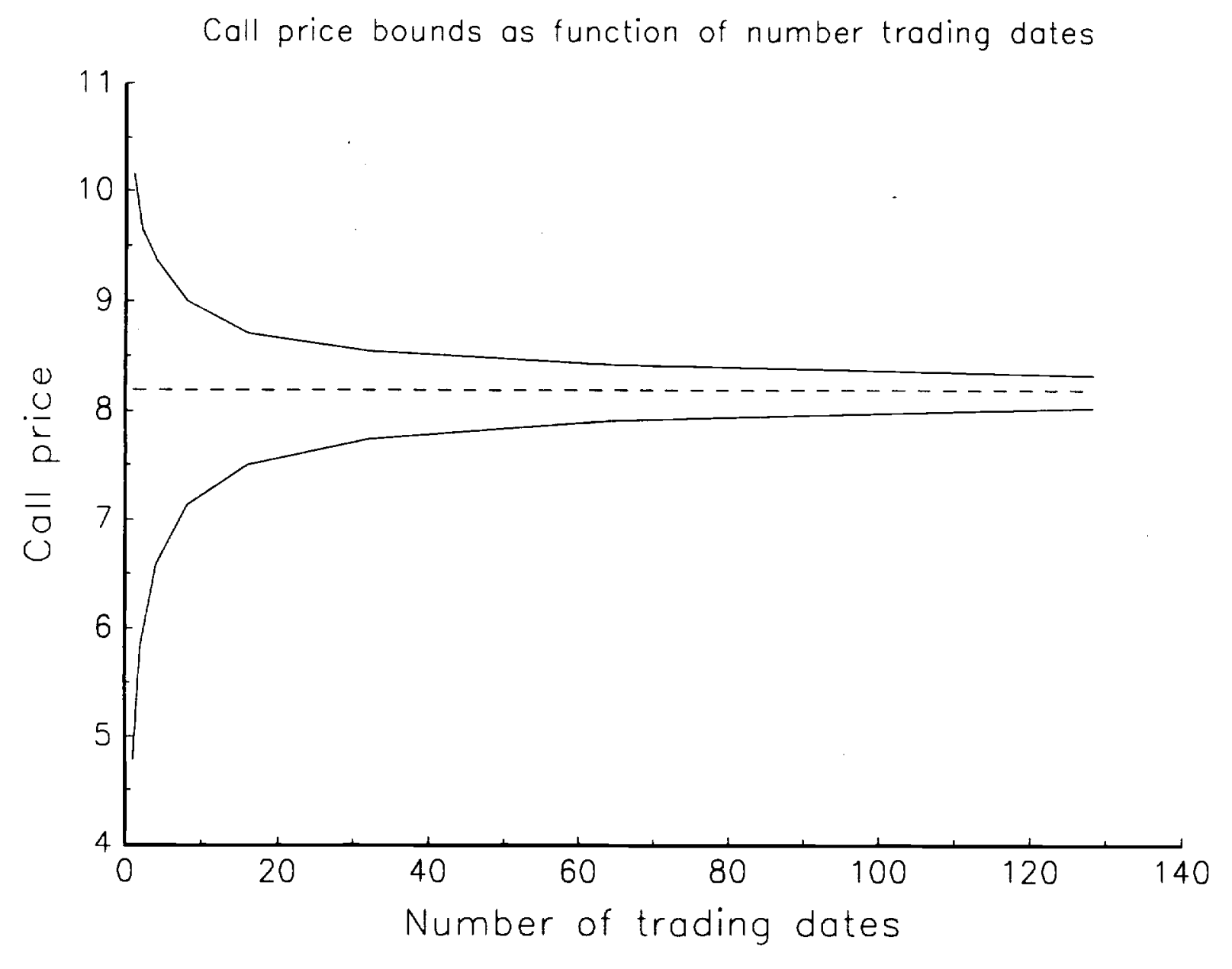

Figure 7: Call price bounds for at-the money option, one year to go, as a function of the number of allowed trading dates. 


\section{Price bounds with stochastic $V$}

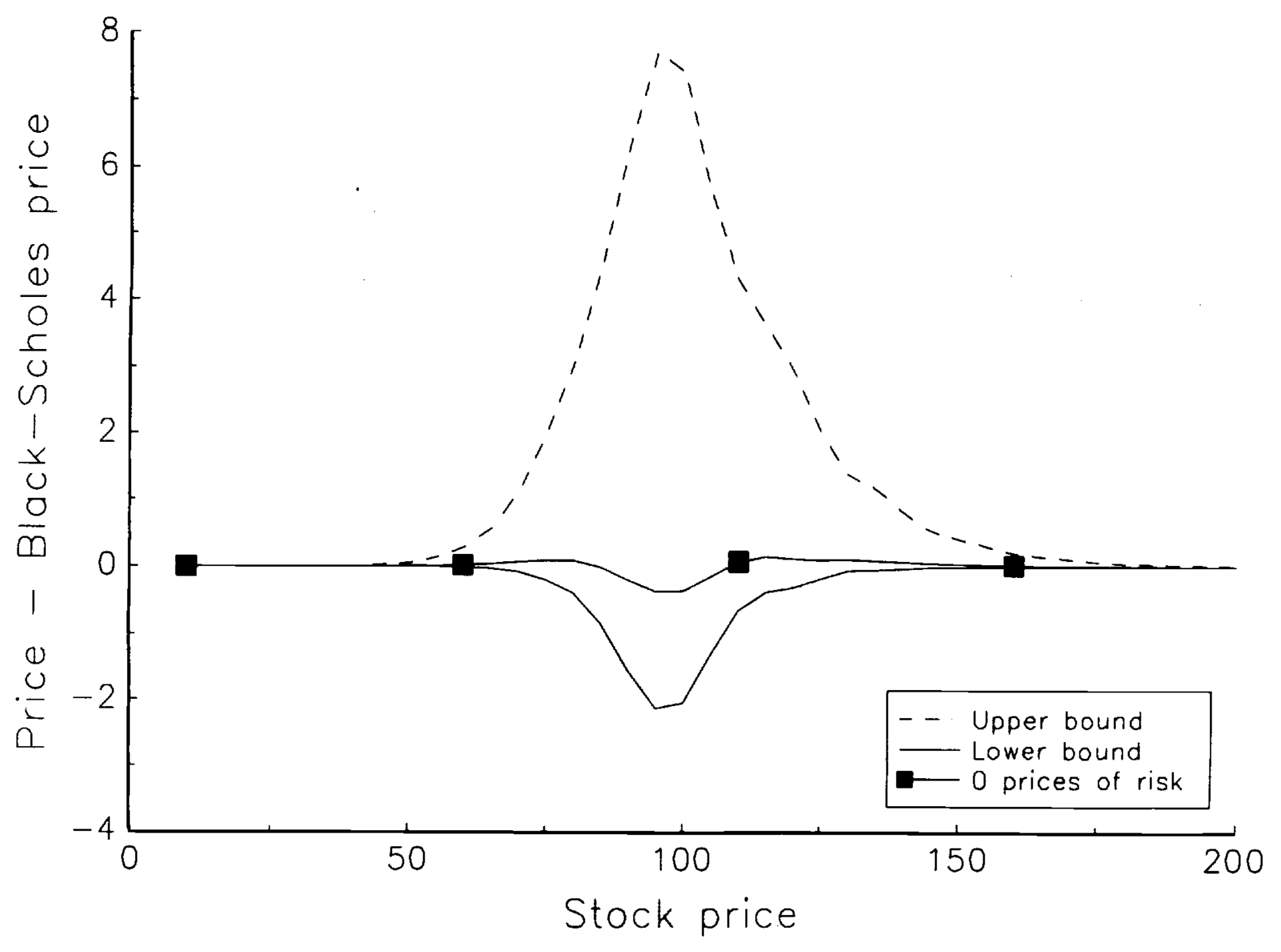

Figure 8: Difference between option price bounds and Black-Scholes price, using stochastic volatility model with constant interest rate. The line in the middle gives the difference between the option price calculated with a zero price of volatility risk and the Black-Sholes price. 


\section{Price bounds with stochastic $V$ and $r$}

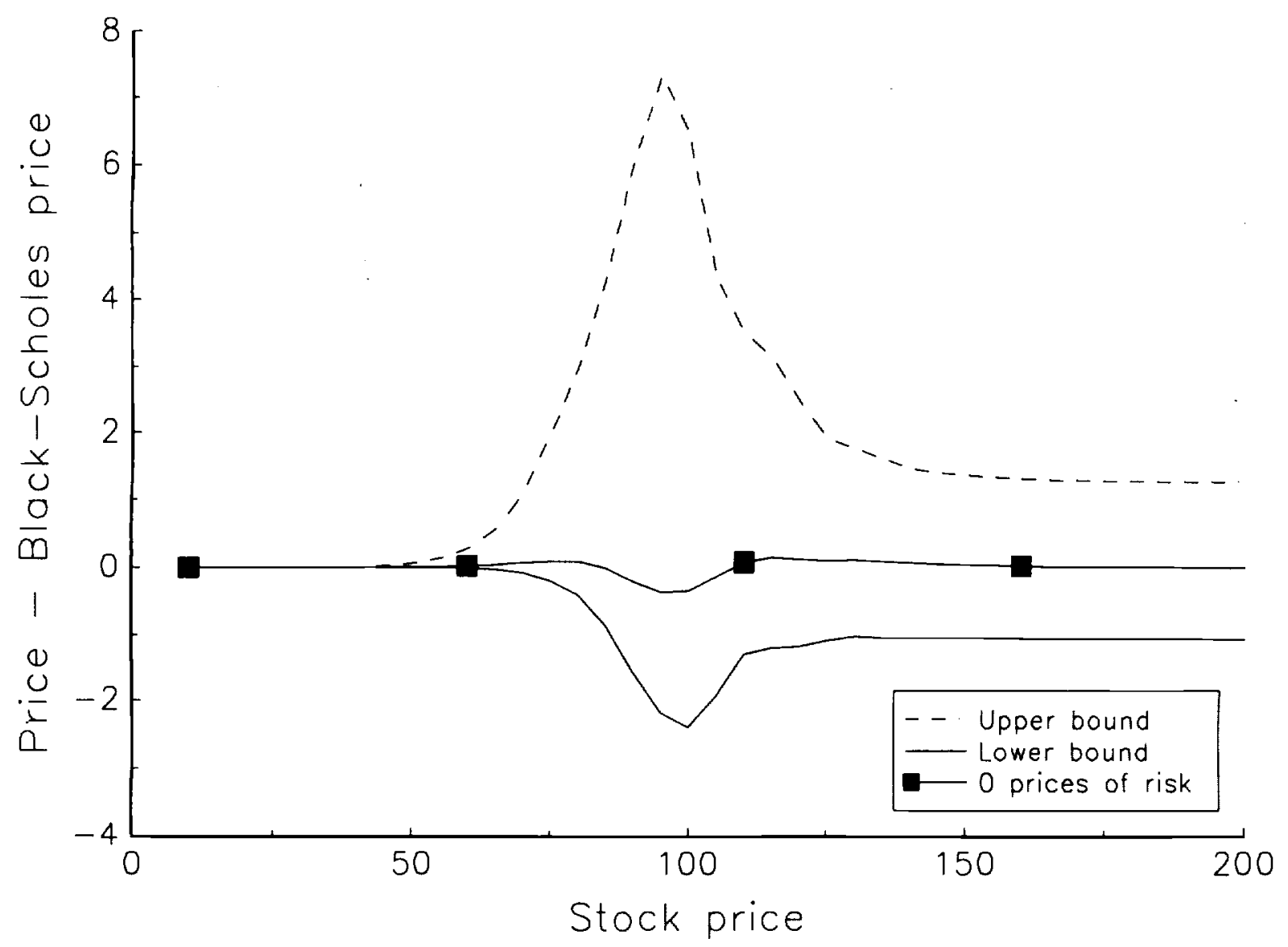

Figure 9: Difference between option price bounds and Black-Scholes price, using stochastic volatility model with stochastic interest rates. The line in the middle gives the difference between the option price calculated with a zero price of volatility and interest rate risk and the Black-Scholes price. 